\section{Pacific Northwest}

National Laboratory

Operated by Battelle for the

U.S. Department of Energy

\title{
Status of Models for Land Surface Spills of Nonaqueous Liquids
}

\author{
C. S. Simmons \\ J. M. Keller
}

August 2003

Prepared for the U.S. Department of Energy under Contract DE-AC06-76RL01830 


\title{
DISCLAIMER
}

This report was prepared as an account of work sponsored by an agency of the United States Government. Neither the United States Government nor any agency thereof, nor Battelle Memorial Institute, nor any of their employees, makes any warranty, express or implied, or assumes any legal liability or responsibility for the accuracy, completeness, or usefulness of any information, apparatus, product, or process disclosed, or represents that its use would not infringe privately owned rights. Reference herein to any specific commercial product, process, or service by trade name, trademark, manufacturer, or otherwise does not necessarily constitute or imply its endorsement, recommendation, or favoring by the United States Government or any agency thereof, or Battelle Memorial Institute. The views and opinions of authors expressed herein do not necessarily state or reflect those of the United States Government or any agency thereof.

\author{
PACIFIC NORTHWEST NATIONAL LABORATORY \\ operated by \\ BATTELLE \\ for the \\ UNITED STATES DEPARTMENT OF ENERGY \\ under Contract DE-AC06-76RL01830
}

This document was printed on recycled paper. 


\title{
Status of Models for Land Surface Spills of Nonaqueous Liquids
}

\author{
C. S. Simmons \\ J. M. Keller
}

August 2003

Prepared for

the U.S. Department of Energy

under Contract DE-AC06-76RLO 1830

Pacific Northwest National Laboratory

Richland, Washington 99352 


\section{Executive Summary}

This report describes the status of models for predicting the spreading behavior of nonaqueous phase liquids (NAPLs) when spilled on land. NAPLs are liquids other than water and are commonly immiscible with water. Examples include diesel fuel, brake fluid, transmission fluid, and many chemical solvents. If there are adequate models available to describe spills, hydrologists can determine how the size of a spill relates to the amount spilled and how long it persists. Further, remote sensors can then be used to detect the presence of a spill on land.

A NAPL spill area can often be detected by remote sensing of its vapors with infrared spectroscopy, for a period after a spill. The opportunity for sensing NAPLs having low volatility, however, may depend on the possibility of detecting directly the liquid phase, or adsorbed material, on the surface of a land spill area. Therefore, a NAPL spill model is useful to predict how large an area is produced by a spill of a certain amount and how long it remains visible for remote sensing.

Models of NAPL spill spreading are used to

- Determine spreading area

- Predict liquid content beneath the land surface following a spill

- Estimate the reduction of surface NAPL by drainage and evaporation

- Estimate the persistence of a surface NAPL spill.

Only one land spill model, a simplified screening type, for NAPL spreading was found after searching the scientific literature. This model was built for the U.S. Environmental Protection Agency. It uses the theory of gravity currents from fluid dynamics to represent spill spreading, and it applies the Green-Ampt sharp front model to describe the concurrent infiltration of NAPL into the ground. This spreading model also considers component-wise evaporation of volatile petroleum liquids, but it does not treat drainage away from the surface after a spill. Predicting the drainage just beneath the surface is a critical capability for determining whether remote sensors can detect the liquid phase of a NAPL near the surface after a spill.

An exact solution to the problem of coupling a gravity current of viscous liquid to its simultaneous infiltration into a porous medium was found. It derives from fluid dynamics and can be used as a paradigm for spill spreading on a flat ideally smooth surface. Test calculations of this gravity current theory revealed that the spreading area is strongly controlled by the release rate of a spill and by the subsurface permeability. A more realistic prediction of spreading under less ideal situations, however, must consider the roughness of the land surface.

This report outlines the processes and mechanisms that control spill mechanics on a porous surface. It also defines physical parameters and lists sources of their values required by mathematical theory for spill phenomena. The key parameters are: 
- Liquid properties: density, viscosity, interfacial tension, and vapor pressure

- Land properties: soil-water retention curve, permeability, surface slope, and roughness.

To build a more general model for NAPL spreading over the land, two existing modeling capabilities for overland flow and multiphase subsurface flow of NAPL must be coupled. The two flow theories that must be mathematically coupled are:

- Saint Venant fluid dynamical equations for overland flow of water over a variable topography

- Multiphase fluid flow equations based on Darcy's law for simultaneous flow of air, water, and NAPL in subsurface porous media (e.g., soils).

The Saint Venant equations for overland flow of water must be modified to account for the different density and viscosity of NAPLs. Simulators for multiphase subsurface flow are highly developed, and many codes are available for application to spill problems. Specific overland flow models and multiphase flow codes that could be employed are identified in this report.

A multiphase subsurface flow simulator is needed when the land is wet to predict NAPL infiltration, because it depends on the pore space not already filled with water. For instance, rainfall on a spill region may displace absorbed NAPL back toward the surface to become visible again. Drainage of NAPL must also be calculated as a three-fluid flow process, because it depends on the concurrent flow of water and air in the subsurface. This property of NAPL immiscibility demands the use of a multiphase flow simulator.

To couple an overland flow model and a multiphase flow simulator for subsurface flow, the height of the surface liquid head must be passed to the subsurface simulator to determine the rate of infiltration. In addition, this infiltration rate must be subtracted from the overland flow volume as liquid sinks in while it spreads. For more volatile NAPLs, which can evaporate during the spill or after, this loss of liquid from the spilled volume must be modeled. Subtracting the infiltration and evaporation from the spilled volume reduces the possible spreading; therefore, these processes of spill loss must be modeled.

The recommendation is to couple an overland flow model with a multiphase subsurface flow model to create a general spill-spreading model, which also accounts for complicated land topography. However, using a general spreading model created by coupling overland and subsurface NAPL flow requires more detailed data than is needed by a simplified spill-screening model. 


\section{Contents}

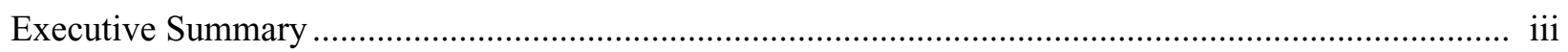

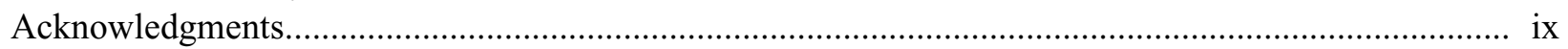

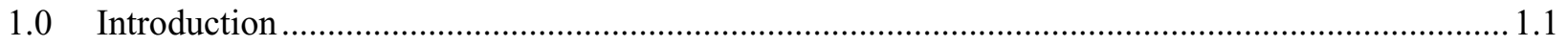

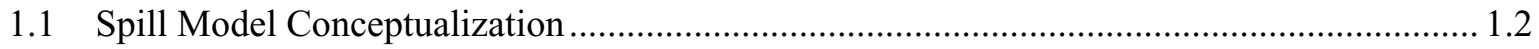

1.2 Spills Versus Leaks and Future Needs ………………………………………………..... 1.4

1.3 Conceptual Submodels of a Spill Model ........................................................................... 1.7

1.3.1 NAPL Release Model................................................................................. 1.7

1.3.2 Overland Flow Model …………………………………………………….... 1.7

1.3.3 Multiphase Subsurface NAPL Flow Simulator....................................................... 1.7

1.3.4 Surface NAPL Concentration Model …………………........................................ 1.8

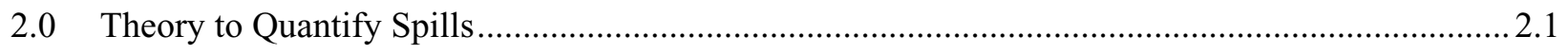

2.1 Infiltration of Nonaqueous Phase Liquids into the Subsurface …………………………...... 2.1

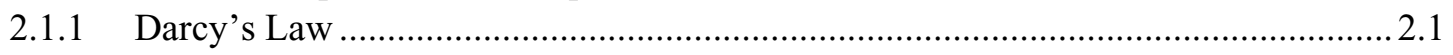

2.1.2 Liquid Retention Relations.............................................................................. 2.2

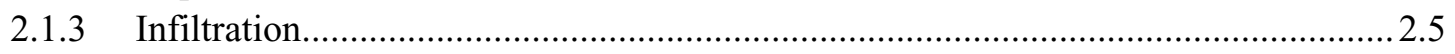

2.1.4 Simplified Infiltration Model ............................................................................... 2.6

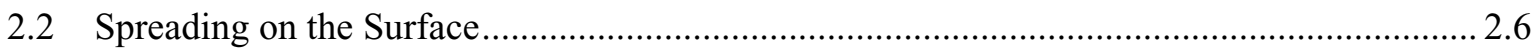

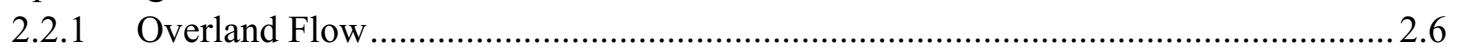

2.2.2 Simplified Spreading Model ……………………………………………..... 2.7

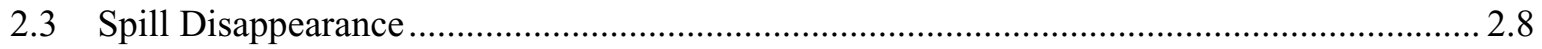

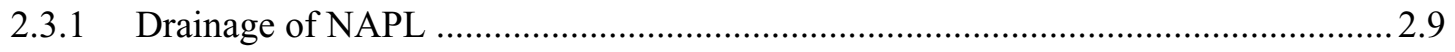

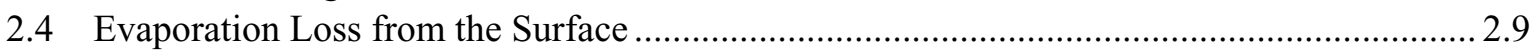

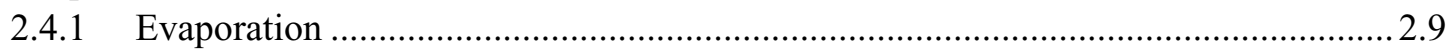

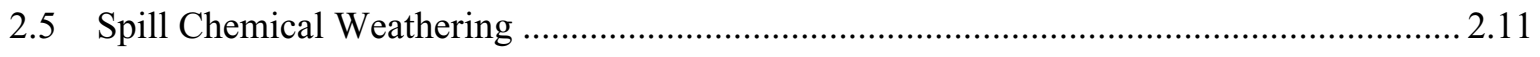

2.5.1 Chemical Transformation.............................................................................. 2.11

2.5.2 Photochemical Transformation ............................................................................. 2.11

2.5.3 Microbial Transformation ............................................................................... 2.12

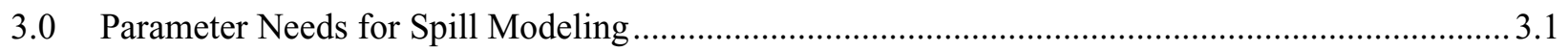

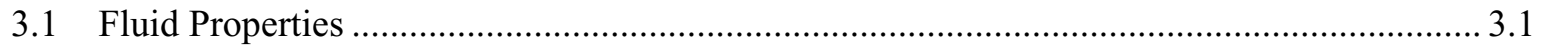

3.1.1 Density of NAPL ...................................................................................... 3.1

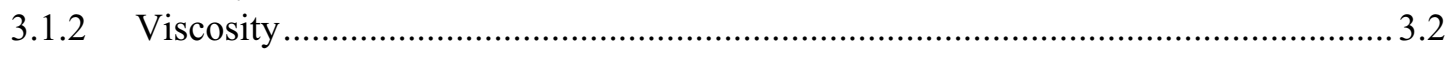

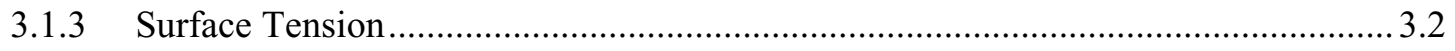

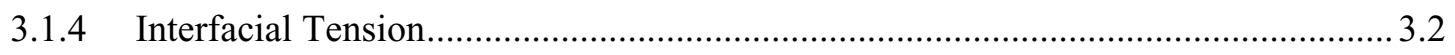

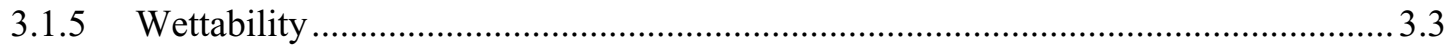

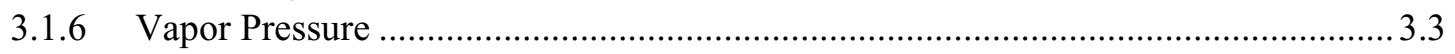

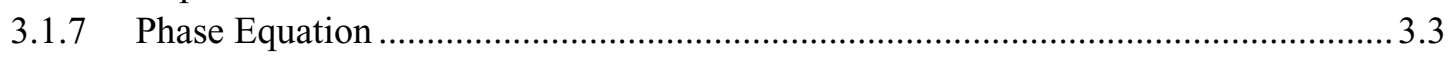

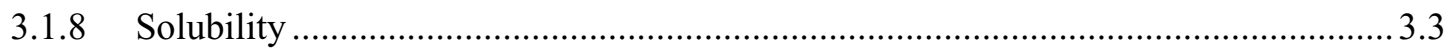

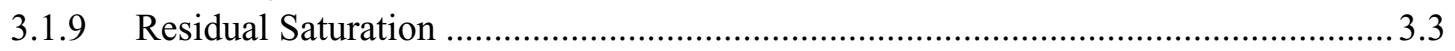

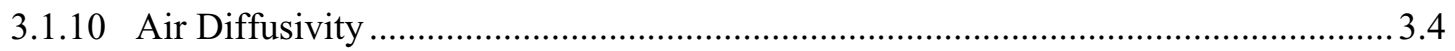

3.1.11 Henry's Constant ................................................................................... 3.4

3.1.12 Degradation Coefficient …………………………………………………..... 3.4 


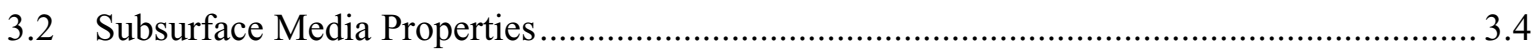

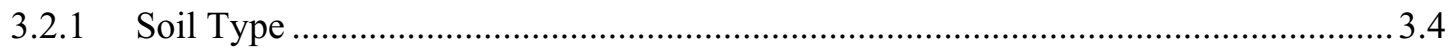

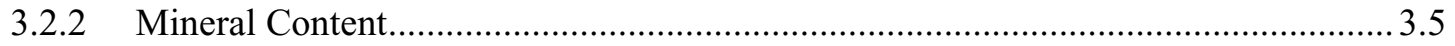

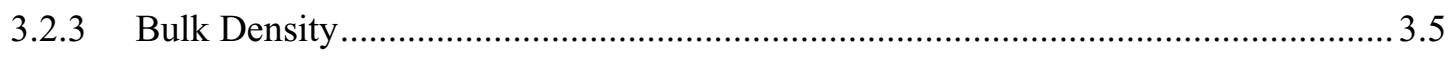

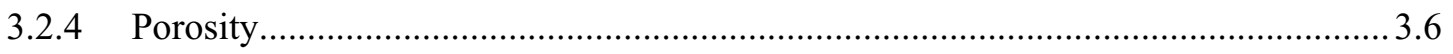

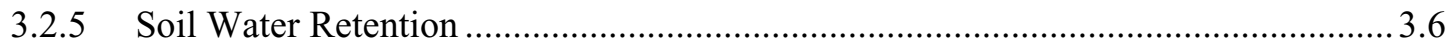

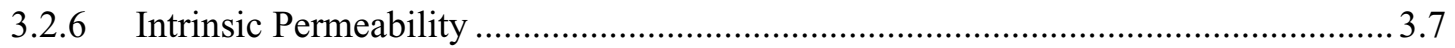

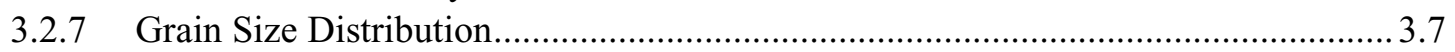

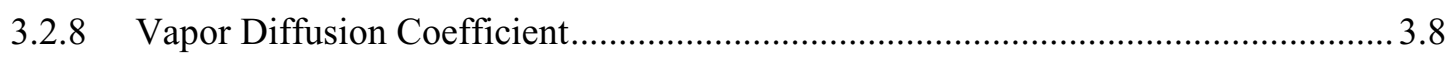

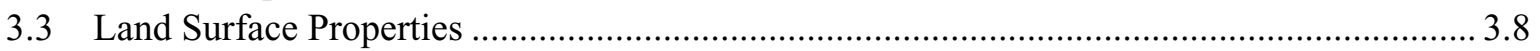

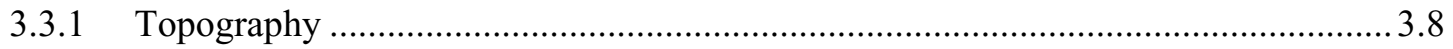

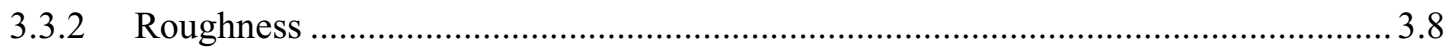

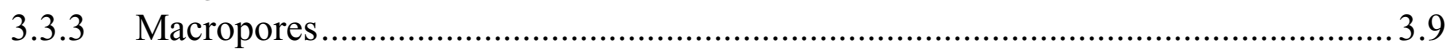

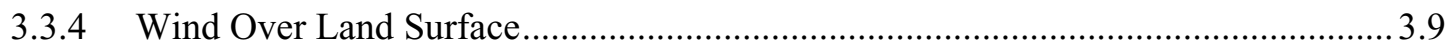

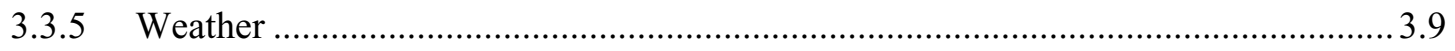

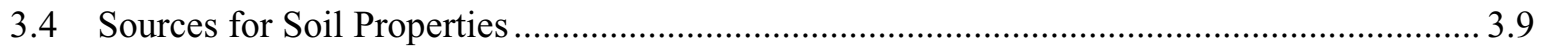

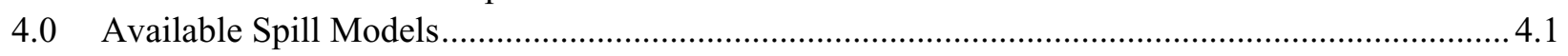

4.1 Screening Model for Surface Spreading ...................................................................... 4.1

4.2 Models Treating Partial Problem Aspects ....................................................................... 4.1

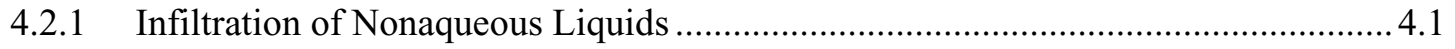

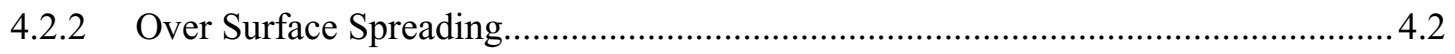

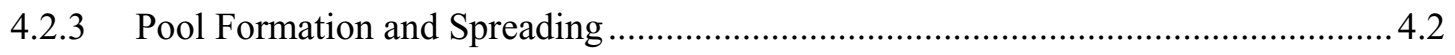

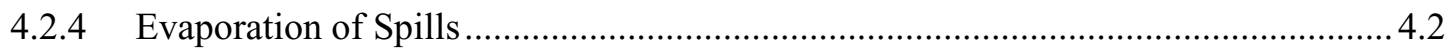

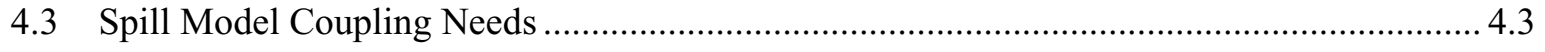

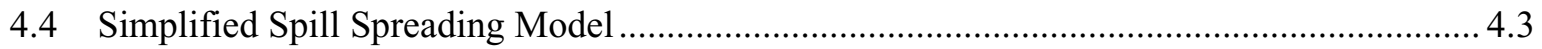

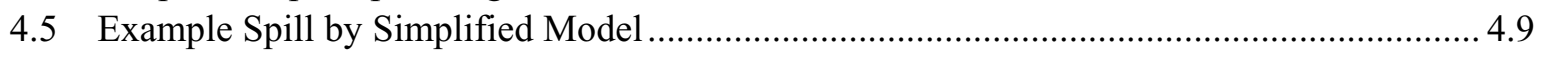

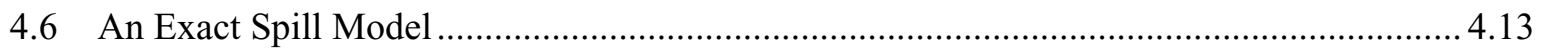

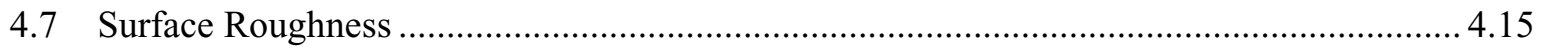

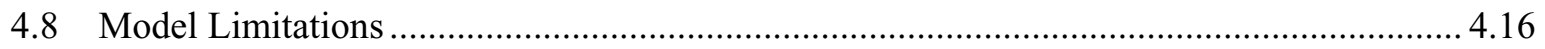

5.0 Conclusions on "State-of-the-Art" of Spill Modeling .............................................................. 5.1

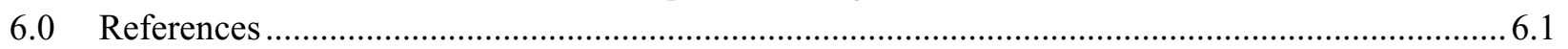




\section{Figures}

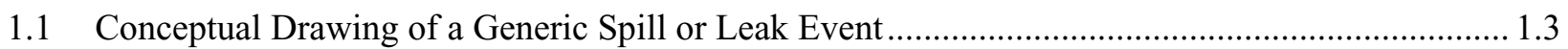

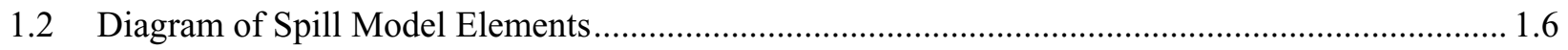

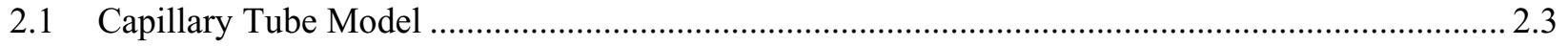

2.2 Air-Water Retention Relations for a Gravel, Sand, and Silt Loam .............................................. 2.5

4.1 Infiltration into Yolo Light Clay Soil under Ponded Condition.................................................. 4.10

4.2 Average Spill Head for a Line Source by Simple Model .............................................................. 4.11

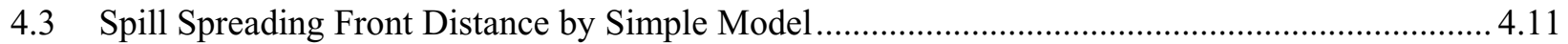

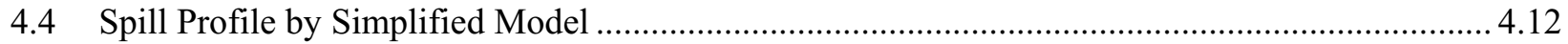

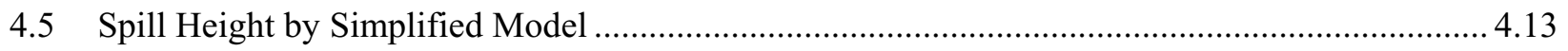

4.6 Spreading and Infiltration of a Spill on a Flat Surface ............................................................ 4.14

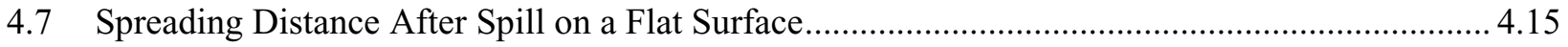

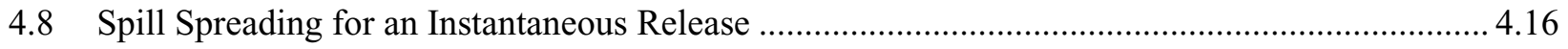

\section{Tables}

1.1 Publications Relevant to Building a Spill Spreading Model and Subject Emphasis ..................... 1.5

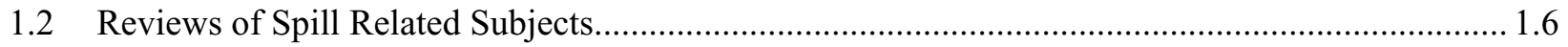

3.1 Spill Model Fluid Parameters, Units, Symbols, and Sources of Information ................................. 3.1

3.2 Spill Model Subsurface Media Parameters, Units, Symbols, and Sources of Information .............. 3.5

3.3 Spill Land Surface Parameters, Units, Symbols, and Sources of Information ............................... 3.8

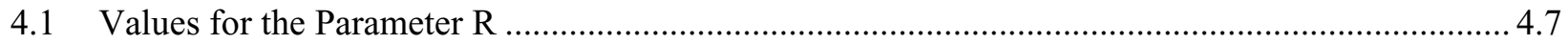




\section{Acknowledgments}

Jeff Hylden is the project manager on this study, and he assisted as peer reviewer. The authors thank him for many readings of the report and his suggestions on improving the content. Kristin Manke is the editor who contributed to streamlining the presentation. 


\subsection{Introduction}

This report discusses models for describing the behavior of nonaqueous phase liquids (NAPLs) spilled over a land surface. A NAPL is a liquid that is not water and usually is a petroleum product or a liquid chemical solvent. For instance, jet fuel, engine oil, benzene and other petroleum products, and solvents such as carbon tetrachloride are NAPLs. These liquids are commonly immiscible with water and constitute a separate liquid phase if they enter the subsurface with groundwater. Liquids such as alcohols, which would mix or dissolve in water, are also spill possibilities but are not dealt with here. This study addresses NAPLs that are not highly volatile and as such would not tend to exhibit a large evaporative mass loss before being mainly absorbed into the land. Examples of those NAPLs include brake and transmission fluids and some fuel oils. Moreover, NAPLs with low volatility may leave residual amounts that remain detectable as a liquid phase just beneath the land surface. A NAPL such as carbon tetrachloride being substantially volatile would present little opportunity for detecting its liquid phase near the land surface after being entirely infiltrated.

The study devises a NAPL spill model that can estimate the spreading area, which possibly would determine the liquid's detectability by remote sensing. A basic question is whether a NAPL of low volatility when spread over the land by a spill can be detected as liquid by remote sensing. If sufficient liquid remains near or just beneath the surface, it may be possible to identify the chemical composition and surface distribution of a NAPL by using remote infrared spectroscopic identification. The size of the surface region wetted by a particular NAPL spill clearly determines the opportunity for detecting it by remote sensing technology. Therefore, the main attribute of a model for treating NAPL spills on a land surface is the capability to predict spreading behavior. However, clearly, how rapidly a spilled liquid disappears into the subsurface determines how far it may spread on the land surface. Thus, the modeling of both liquid spreading and infiltration into the subsurface are equally important to determining the extent of a spill.

The modeling of NAPL spill spreading on a land surface is not limited to non-volatile liquids because of any limitation in the physical theory relevant to predicting the spreading. Non-volatile liquids are mainly of interest because their liquid phase may remain detectable just beneath the land surface by using remote sensing. The feasibility of NAPL spill detection requires consideration in terms of its disappearance behavior after spreading. Therefore, this study considers the importance of evaporation as it influences spill spreading.

Usually, spill models are conceived to deal with the spill of petroleum on water during transportation. A recent state-of-the-art review of oil spill modeling was accomplished by the American Society of Civil Engineers (1996), in which they considered typical processes that govern the fate of a spill on water. The review is germane because many of these same processes are relevant to oil spilled on land.

In extreme contrast to spills on water, spills of NAPL on land usually are absorbed into the subsurface, assuming the spilled substance does not first evaporate nearly entirely into the atmosphere. The extent to which absorption or infiltration of a NAPL into the subsurface occurs depends strongly on the character of the subsurface porous material: sand, clay, gravel, rock, or pavement. Geological and soil type attributes as well as the amount of water already stored in the subsurface control how rapidly a 
NAPL disappears from sight on the surface. The concentration of NAPL remaining near the surface naturally determines if it can be detected by means of direct infrared spectroscopy, foregoing the need to obtain a subsurface sample for laboratory analysis.

Because hydrology deals with the movement of liquids (usually water) over the land surface and into the subsurface, this subject area was extensively searched first for physical theory and models relevant to the stated modeling objectives. Other areas such as fluid mechanics and chemical engineering were also sources of relevant theory for treating spill propagation and fate. Multiphase fluid flow capable of describing the movement of NAPL in the subsurface is a highly developed branch of hydrology with many computer codes available for treating the movement of NAPL in the ground. Much of this theory was originally derived from petroleum engineering needs to recover oil. The theory has since evolved into addressing groundwater contamination caused by NAPL. Miller et al. (1998), for instance, give an up-to-date review of the multiphase flow theory required to describe the subsurface migration of NAPL. Appropriate computer codes for solving the theoretical equations of all degrees of generality are identified in that extensive review. Multiphase flow theory is required because it treats problems involving the three phases (gas, water, and NAPL) in a porous medium. NAPL evaporation into the gas phase is addressed as well.

In contrast to the highly developed capability for treating subsurface flow of NAPL, this review finds that surface hydrology, dealing commonly with overland flow of water, has not extensively addressed the aspect of overland flow of NAPLs, as is required to delineate spills. However, the physical theory of overland flow of water can be readily modified by taking into account the physical differences in density and viscosity of a NAPL. The physical theory of overland flow is based on basic fluid dynamics with application to complicated topography, as is needed to describe the variable earth surface at whatever spatial scale is appropriate for a particular spill situation.

\subsection{Spill Model Conceptualization}

A spill conceptualization guides what mathematical theory of liquid flow is likely required to describe the particular situation. Figure 1.1 is a conceptualization. The phenomenological theory for the processes that are involved also indicates what physical parameters or attributes of the system (land surface and subsurface) are needed to quantify the potential dispersal behavior of a NAPL. A later section lists the required parameters after discussing basic theory.

Aspects that control spreading of a spill are the following:

1. How much liquid is spilled (amount).

2. How rapidly the liquid is released (rate).

3. How much liquid the porous subsurface can absorb (infiltration).

4. Slope of the land topography (dispersal direction on surface). 


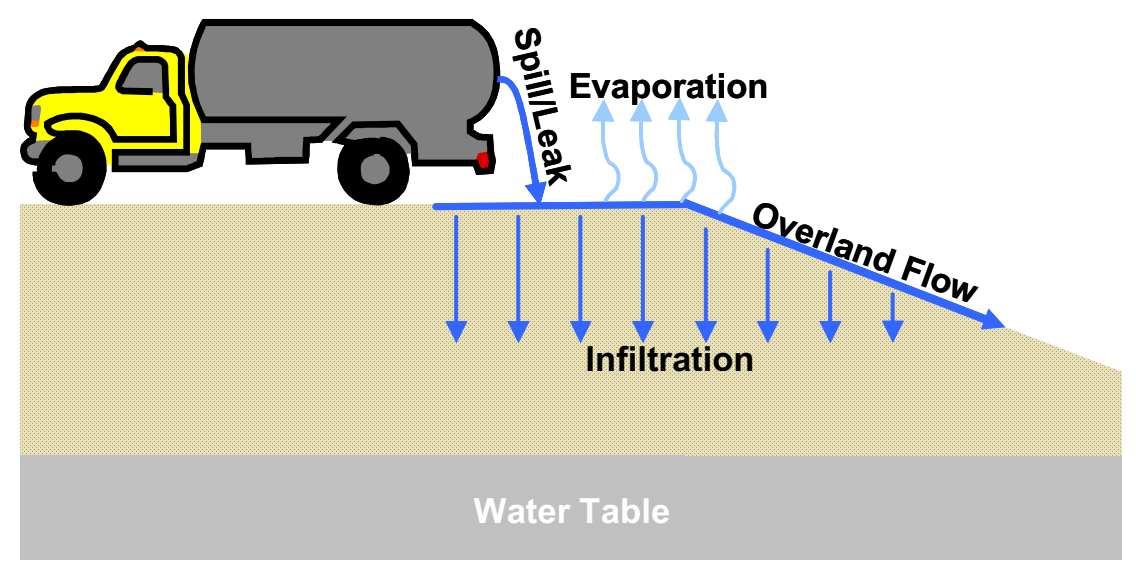

Figure 1.1. Conceptual Drawing of a Generic Spill or Leak Event

A spill of liquid might occur as a gradual release from a container onto the land, or by way of a catastrophic abrupt release of a liquid wave. Intuitively, one knows that how much liquid spreading may occur on a surface depends on how rapidly the liquid is released. This is especially so for a spill on a porous surface with either a limited or unlimited capacity to absorb the liquid. A subsurface with a limited absorption capacity might have an impermeable layer just below the surface. How far a liquid travels over the surface and its eventual distribution also depends considerably on the land topography. A basic example is the influence of gravity when a liquid is spilled on a flat level surface as opposed to an incline. On a slope, the running liquid may form rivulets that do not wet the entire surface.

How far a spill spreads is greatly controlled by infiltration, as well as by the overland flow dynamics. A liquid spill changes depth or height as it moves over the terrain. That changing depth controls infiltration. How much terrain is inundated depends generally on the spill amount but is limited by the infiltration. In the usual three-phase flow situation, for which the subsurface is not saturated with either NAPL or water, the liquid head (i.e., the depth of the spilled NAPL at each location) and capillary pressures determine how strongly the infiltration occurs. The rate of infiltration also depends on the viscosity of the NAPL, as well as on the water content of the porous subsurface. The physical nature of the subsurface medium (soil texture, for instance) also influences the infiltration. Coarse sand, tight clay, and fractured rock all constitute a different medium, able to absorb different amounts of spill. A spill that physically cannot be imbibed must continue to run over the land surface or eventually form standing pools.

Finally, during and after the spill, some of the NAPL may evaporate depending on its volatility and environmental conditions. This aspect of a spill model requires special additional theory to describe the longer-term disappearance of surface NAPL as a result of many weathering or environmental processes. These processes are briefly discussed in later sections. 


\subsection{Spills Versus Leaks and Future Needs}

Because of the disparity in the development of science to address flow of NAPL on the land surface and in the subsurface, this search was not able to identify more than a few models designed to deal explicitly with land spreading of NAPL spills. What is lacking is a concerted effort by researchers and model developers to couple the processes of surface flow and subsurface flow of NAPLs. Future models, which deal with equations of surface flow and subsurface flow on an equal footing, have to couple the mathematics to treat the connection of overland and subsurface flow. Because of this disparity in capability, it is expedient and convenient to divide spills into two main categories: leaks and spills. Each of which occurs over some defined time period.

A leak of NAPL is defined here to mean a well-defined entry of liquid into the subsurface porous medium (in time and place). Multiphase flow theory can deal with problems for which the leak area and rate of entry are well specified. The nature of the subsurface porous media involved and properties of the NAPL are all that is required to predict the subsurface flow behavior. There remain many scientific challenges to making accurate predictions of subsurface multiphase flow. Those challenges are discussed by Miller et al. (1998) and are indicated in later sections of this report.

A spill of NAPL involves the overland flow as well as the concurrent infiltration of the liquid. During a spill, the amount and duration must be specified to make a well-specified problem. In a spill problem, the area covered by the liquid is to be determined from coupling the mechanisms of overland and porous media flow. A true spill model is intended to predict the area of dispersal and the NAPL concentration that remains near the surface as time passes. For purposes of infrared signature detection, the prediction of the surface concentration, or NAPL content, may have to be very accurate to identify the spill region. In a spill situation, the release rate of NAPL or the input must be specified to make the problem defined and have a mathematical solution. In contrast, in a leak problem, the NAPL input to the subsurface is presumed specified as a boundary condition.

Models for NAPL spills may be complex or simple depending on the technical detail used to evaluate or predict the liquid movement. The difficulty with using a spill model is making it consistent with the pertinent physical details that control the situation, such as incorporation of the detailed information (parameters) for describing the interaction of liquid physics and the land stratigraphy. Available spill models, which are a simplified screening type, can at best make a relative comparison of the various process mechanisms and conditions under which a spill occurs. This search was only able to find one simplified screening surface spill model (Hussein, Jin, and Weaver 2002). The model was designed to explicitly treat surface spreading of petroleum and to serve the purposes of the U.S. Environmental Protection Agency, such as an environmental assessment of how much petroleum product could eventually end up in the groundwater. The spill model attempts to incorporate all physical mechanisms that could conceptually influence the long-term impact of a spill. It specifically incorporates overland spreading, infiltration, and evaporation of volatile petroleum components in a coupled way. The model is considered a simplified screening type because every component submodel is a highly simplified solution of the mathematical theory expected to describe the physical processes involved. Indeed, the component models are at best only approximate solutions to the exact governing physical theory. In other words, the model cannot be expected to be an accurate predictor of how a NAPL spill would actually end up being 
distributed over the land surface. Moreover, the model does not estimate the NAPL content remaining on the surface over time as an amount detectable, because it does not address long-term drainage. Long-term drainage by which NAPL continues to sink deeper determines what liquid content would be detectable at the surface over time.

Table 1.1 summarizes the situation found for models that could be used to create a land surface spill model. Table 1.2 provides selected references that address the subjects that were found to be essential for understanding spill phenomena. Figure 1.2 indicates the needed linkage of models given in Table 1.1.

Because a list of existing land spill models having a range of simplicity or complexity could not be located in the hydrology, environmental engineering, or fluid dynamics literature, the following sections of this report address what is likely required in terms of physical theory, parameters, and modeling conceptualization to build a future spill model. Fortunately, due to the extensive development of theory regarding overland water flow and subsurface flow of NAPL, the development of a spill model would only require a rigorous coupling of the equations describing overland and subsurface flow of a NAPL. Such coupling requires a return of predicted information from the subsurface model back to the overland flow model. Other aspect of the spill modeling procedure can be accomplished in a sequential forward passage of predicted information. The plan of a spill model given in Figure 1.2 determines this sequence of modeling steps.

Table 1.1. Publications Relevant to Building a Spill Spreading Model and Subject Emphasis. Any subsurface model could be coupled to an overland model to create a spill model.

\begin{tabular}{|c|c|c|c|c|}
\hline \multicolumn{2}{|c|}{ "Overland Flow } & \multicolumn{2}{|c|}{ Subsurface Flow } & \multirow[t]{2}{*}{ Publication } \\
\hline Gravity Current & $\begin{array}{l}\text { Saint Venant } \\
\text { Equations }\end{array}$ & Infiltration & Drainage & \\
\hline Yes & & NA & NA & $\begin{array}{l}\text { Huppert (1982) } \\
\text { Fluid Dynamics }\end{array}$ \\
\hline Yes & & Green-Ampt NAPL & No & $\begin{array}{l}\text { Acton et al. (2001) } \\
\text { Fluid Dynamics }\end{array}$ \\
\hline Yes & & Green-Ampt NAPL & No & $\begin{array}{l}\text { Hussein et al. (2002) } \\
\text { Screening Model }\end{array}$ \\
\hline NA & NA & Green-Ampt NAPL & Yes & $\begin{array}{l}\text { Reible et al. (1990) } \\
\text { Simplified Model }\end{array}$ \\
\hline \multirow[t]{3}{*}{ NA } & NA & Green-Ampt NAPL & No & \begin{tabular}{|l|} 
Weaver et al. (1994) \\
Simplified Model
\end{tabular} \\
\hline & Yes & Green-Ampt Water & No & $\begin{array}{l}\text { Esteves et al. (2000) } \\
\text { Surface Hydrology Model }\end{array}$ \\
\hline & Yes & Green-Ampt Water & No & $\begin{array}{l}\text { Leonard et al. (2001) } \\
\text { Surface Hydrology Model }\end{array}$ \\
\hline NA & NA & $\begin{array}{l}\text { Yes } \\
\text { NAPL and Water }\end{array}$ & $\begin{array}{l}\text { Yes } \\
\text { NAPL and Water }\end{array}$ & $\begin{array}{l}\text { Kaluarachchi and Parker (1989) } \\
\text { Multiphase Flow Simulator }\end{array}$ \\
\hline NA & NA & $\begin{array}{l}\text { Yes } \\
\text { NAPL and Water }\end{array}$ & \begin{tabular}{|l} 
Yes \\
NAPL and Water
\end{tabular} & $\begin{array}{l}\text { White and Oostrom (2000) } \\
\text { Multiphase Flow and Transport } \\
\text { Simulator }\end{array}$ \\
\hline
\end{tabular}


Table 1.2. Reviews of Spill Related Subjects

\begin{tabular}{|l|l|}
\hline \multicolumn{1}{|c|}{ Publication } & \multicolumn{1}{c|}{ Spill Related Subjects } \\
\hline ASCE (1995) & Oil spills on sea \\
\hline Fingas (1995) & Spill evaporation \\
\hline Mackay and Matsugu (1973) & Hydrocarbon evaporation \\
\hline Van Den Berg et al. (1999) & Pesticide evaporation \\
\hline Yates et al. (2002) & Pesticide volatilization \\
\hline Mercer and Cohen (1990) & Review of multiphase subsurface flow behavior \\
\hline Miller et al. (1998) & Review of multiphase subsurface models \\
\hline Mohanty et al. (2002) & Soils property database \\
\hline Nemes et al. (2001) & Soils database \\
\hline ASCE = American Society of Civil Engineers. \\
\hline
\end{tabular}
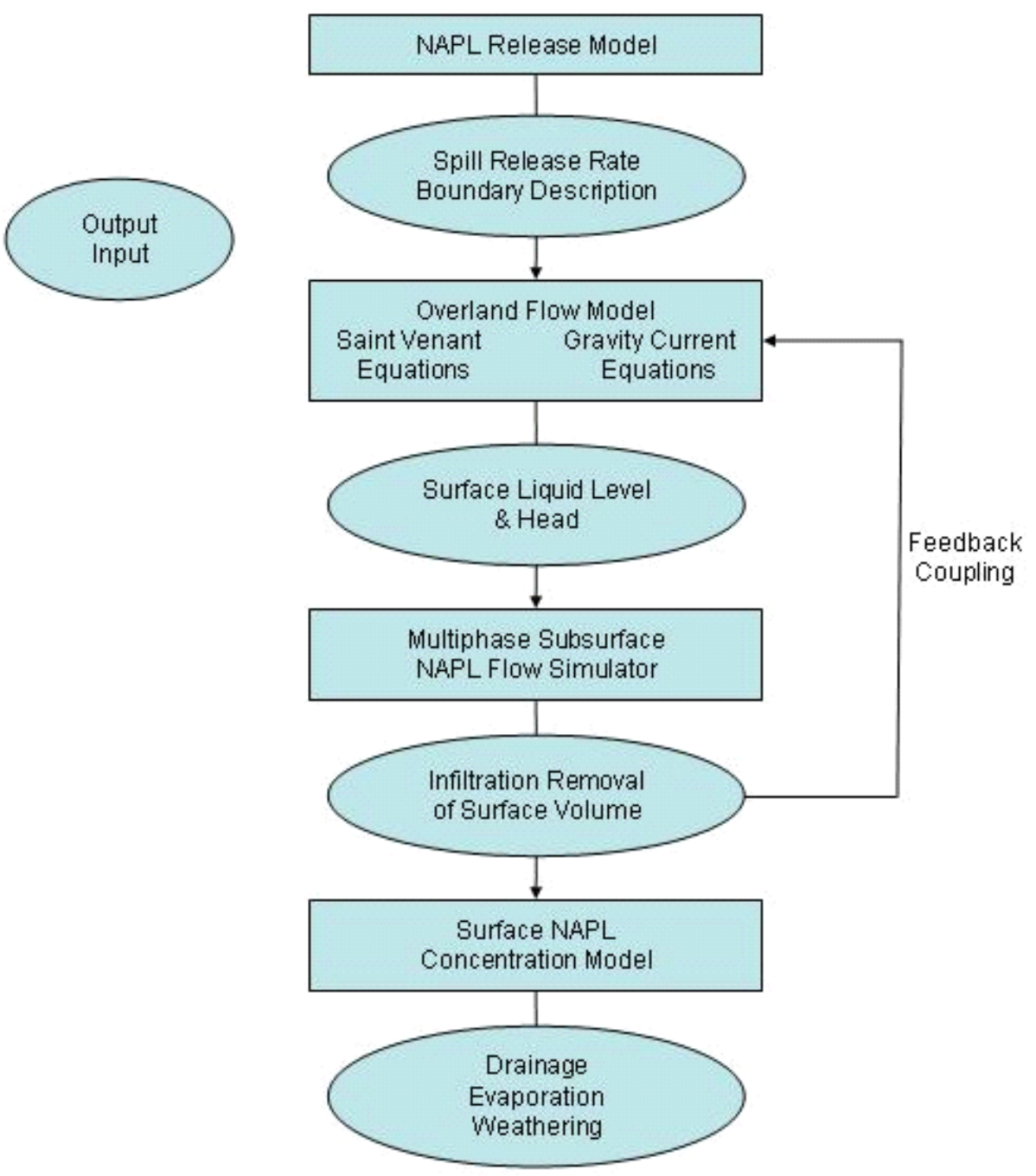

Figure 1.2. Diagram of Spill Model Elements 


\subsection{Conceptual Submodels of a Spill Model}

Each process depicted in Figure 1.2 requires a model (submodel) that describes the physics of the process. Each model would need to be coupled mathematically to constitute an entire spill/leak model.

\subsubsection{NAPL Release Model}

The first submodel describes the release rate or amount per unit time of a particular liquid being spilled. If the release pattern and spill boundary region (i.e., spill area) of soil entry are known precisely, or presumed so, this event would be called a "leak" situation. Recall that in a leak scenario, the spreading area is presumed specified. A general subsurface multiphase flow simulator could then be used to describe the vanishing of the liquid into the ground. More generally, for a spill situation, the overland flow area and runoff behavior are going to be unknown and must be predicted based on the particular release model. In a spill situation, the release model, which provides the input to the overland flow, must be specified as a boundary condition to specify the problem solution. In particular, conceptually, the amount of spatial spreading that occurs is going to depend on the input region where the spill meets the ground and on the liquid's release rate. Intuitively, a faster release should produce a greater tendency to spread, because there is less time for the process of infiltration to act on the pool near the source. So for a spill problem an exact mathematical description of how the released liquid is input to the overland flow model must be provided. A considerable variety of release models might be needed to describe many different ways a spill occurs: tank hole, tank rupture, pipe drip or leak, and so forth.

\subsubsection{Overland Flow Model}

A model based on equations for overland movement of a viscous liquid is required to predict spreading, possibly in three dimensions. A simplified one-dimensional description may be adequate to provide insights into the relative spreading compared with the infiltration. The equations for gravity currents (Acton et al. 2001) or the Saint Venant equations (Esteves et al. 2000) for depth-averaged overland flow could be used to describe this part of the problem. Gravity currents can be used to describe spreading on flat perfectly smooth surfaces, which might be inclined uniformly, but the Saint Venant equations would be needed for large flow regions with spreading over variable topography. The complexity of the model depends on the intended prediction accuracy and on the availability of information about the land surface: hydraulic properties and topography, for instance.

\subsubsection{Multiphase Subsurface NAPL Flow Simulator}

A physical model is required to describe how quickly liquid enters the subsurface as it moves over the land surface. This third model box of Figure 1.2 is essentially the model for infiltration of NAPL. The multiphase flow simulator is not actually a model until a great deal is specified about the boundary conditions and distribution of porous properties of the subsurface. It is the liquid that remains just below the surface, after a spill has sunk in, that will likely be detectable by infrared scanning. In general, a multiphase subsurface NAPL flow simulator is needed to treat the infiltration process that depends on the overland flow. In general, under the most complicated of conditions, both air and water will be present and control the infiltration of a NAPL. The entry of liquid into the subsurface could be described by a simple model such as the Green-Ampt equation, appropriately modified to describe a NAPL. Hussein 
et al. (2002) describe a particular approach for coupling the overland flow and infiltration models. In general, the infiltration submodel, however, would need to be as precise or accurate as the capability included in the Subsurface Transport Over Multiple Phases (STOMP) simulator (White and Oostrom 2000), which solves the general three-phase flow problem in a heterogeneous layered three-dimensional subsurface medium. Which model would be used depends on the available fluid and porous media data and the particular predictive accuracy that is required.

The infiltration model, a subsurface flow simulator, might also incorporate a representation of the drainage stage during which the absorbed liquid content declines at the surface as liquid continues to move downward into the subsurface. Drainage, however, does not begin until the supply of liquid on the surface stops. That is, there is no need to model the drainage stage until the surface liquid has sunk in. Note that although the spilled liquid may have sunk in and vanished from the surface, it might not have disappeared from sight or detection just below the surface. Drainage and evaporation, and additionally weathering, determine when the spilled liquid actually, finally, disappears. It is expedient and convenient to include drainage in the last submodel that describes processes that lead to the disappearance of liquid from just beneath the surface.

\subsubsection{Surface NAPL Concentration Model}

This submodel is intended to describe the changing NAPL concentration (content) near the land surface after the spreading has ceased. Evaporation is to be described in this last submodel (last box of Figure 1.2). During and after the spill has occurred, the NAPL remaining near the surface may evaporate or weather away depending on its particular chemical composition and volatility. Many NAPL products are composed of multiple volatile components that evaporate at different rates depending on temperature and air flow rate over the spill surface. How rapidly the NAPL vapor can diffuse back out of the porous subsurface controls how long the NAPL may remain detectable. Under usual circumstances, an evaporation model is assumed sufficient to estimate the long-term progress (i.e., fate) of a surface spill. Evaporation and drainage, however, are coupled processes and act together to alter the surface NAPL concentration. For a low volatility NAPL, evaporation might be negligible and then drainage would mainly control disappearance from the surface. However, for highly volatile liquids, evaporation could cause the dominant flux of liquid from the surface. Note that drainage determines the surface depth over which diffusive flux or evaporation can take place.

Another possibility is that the land surface, say for instance a roadway of asphalt or concrete, is essentially impermeable and flat so that the spill pools on the surface without much absorption. In this case, the evaporation would entirely determine the fate of a spill.

Each model in Figure 1.2 calls for certain physical and chemical parameters required to apply an appropriate mathematical theory. Later sections identify the likely parameters and suggest sources of measurements.

The feedback coupling between overland flow and subsurface flow models indicated in Figure 1.2 is the lacking part. Development of a spill model requires implementing appropriate simulation techniques 
to deal with the coupling. A true spill model cannot be developed unless a method for dealing with the moment-to-moment withdrawal of liquid from the overland flow by the subsurface infiltration is incorporated.

It is recommended that the overland flow theory described by the Saint Venant equations, as modified to account for NAPL viscosity, needs to be coupled with a general multiphase flow simulator for NAPL infiltration into the subsurface. The subsurface NAPL flow code used should have the capability found in the STOMP code (White and Oostrom 2000). Any other multiphase flow code or simulator, however, could be used, such as those reported by Miller et al. (1998). The choice, however, should depend on the user's familiarity for accomplishing a successful application to a spill problem. 


\subsection{Theory to Quantify Spills}

This section describes the three general areas of theory required to quantify the conceptualization of a spill situation, as outlined in Section 1.0. Typically, a spill involves the release of a NAPL, followed by simultaneous overland flow and infiltration into the subsurface, and finally the liquid being diminished or degraded by evaporation and chemical weathering. Drainage also causes the liquid to move further down away from the surface. Evaporative loss might also occur concurrently with the spill release, depending on the liquid's volatility.

This discussion does not attempt to review or repeat mathematical formulations, which are readily available in many technical references, which are referenced as needed. Instead, this section dwells on an explanation of what the theory means. The mathematical theory behind the fluid dynamics pertinent to overland and subsurface flow of a NAPL is technical, and an adequate explanation of the details is not attempted here. Instead, the discussion is aimed at emphasizing behavior of NAPL spills as would be visible near the land surface, as influenced by processes acting below the surface. Many subsurface mechanisms contributing to NAPL dispersal are not considered in this report, but are extensively discussed in the cited reviews of subsurface multiphase flow theory (see Table 1.2).

A spill usually begins with overland flow as the controlling process. However, the infiltration process as determined by the subsurface medium is just as important in determining how far a spill spreads. Moreover, because the theory of NAPL movement in the subsurface is the most mathematically developed and because many computer codes already exist to apply the theory, infiltration is discussed first. Furthermore, a leak situation for which the surface boundary conditions are presumed known can be treated entirely by subsurface flow theory.

\subsection{Infiltration of Nonaqueous Phase Liquids into the Subsurface}

The multiphase flow of a water-immiscible liquid in a porous medium describes the subsurface movement of a NAPL spill. The relevant theory was extensively reviewed by Mercer and Cohen (1990). The main physical and chemical processes relevant to the fate of NAPL in the subsurface are: infiltration, drainage, redistribution, dissolution, interphase mass transfer, soluble transport, and gas phase advectivediffusive transport. For a leak situation, infiltration and drainage are the primary processes of concern.

\subsubsection{Darcy's Law}

Darcy's law describes or quantifies the advective flux of a fluid and defines the movement. A onedimensional form of Darcy's law is:

$$
\mathrm{q}=\mathrm{K} \frac{\Delta \mathrm{P}}{\mathrm{L}}
$$


where $q=$ flux

$$
\begin{aligned}
K & =\text { fluid conductivity } \\
\Delta P & =\text { pressure difference between two points separated by length } L .
\end{aligned}
$$

The pressure gradient, $\Delta P / L$, represents the forces that drive the fluid between spatial locations. In a liquid system, gravity (weight) and capillarity are the primary forces. In a gas system, barometric pressure differences generally drive gas transport.

The fluid conductivity determines how rapidly the fluid flows in response to the associated pressure gradient. Fluid conductivity may be separated into a permeability term $(k)$ and a fluidity term $(f)$ such that:

$$
\mathrm{K}=\mathrm{kf}
$$

Permeability describes the pore space geometry interconnections available for flow through a medium. In a multi-fluid system, the permeability of each fluid is dependent on the fraction of the total pore space it occupies. Fluidity is defined as:

$$
\mathrm{f}=\frac{\rho g}{\eta}
$$

where $\rho=$ fluid density

$$
\begin{aligned}
& g=\text { gravitational acceleration } \\
& \eta=\text { fluid viscosity. }
\end{aligned}
$$

Equation (2.3) shows that fluid conductivity is proportional to permeability and fluid density while inversely related to fluid viscosity.

Three expressions of Darcy's law would be needed to describe the movement and advective flux of each fluid phase (water, NAPL, gas) present. Darcy's law is combined with a continuity (mass conservation) equation for each fluid to determine a set of three motion equations.

Darcy's law accounts for momentum dissipation in each fluid caused by the viscous drag when moving through the interconnected pore space (interstices of the medium). This is why the law when combined with the conservation of fluid mass equation determines an equation of fluid motion.

\subsubsection{Liquid Retention Relations}

Capillary forces act on liquids whenever the porous medium is not entirely saturated. The capillary forces may serve to draw water or NAPL into the interstices or the pore channels of a porous medium. A simplified description of the capillary forces experienced in a pore channel and the pressure differences 
between the non-wetting and wetting phase fluids, called the capillary pressure, may be described by a capillary tube model as shown in Figure 2.1. One can visualize a porous medium as a size-distributed bundle of capillary tubes. Only the smallest radii tubes conduct water to the greater heights. The capillary rise height indicates how strongly liquid is absorbed either up or downward into a porous medium. It is a direct measurement of the relationship between liquid content and the capillary pressure, or suction, that pulls liquid into a porous medium.

Generally, water is the wetting phase and is drawn into a porous medium with greater adhesive force than a non-wetting phase such as NAPL. A positive pressure must be applied to a non-wetting phase to force it into a porous medium already saturated with water. Likewise, water is imbibed into a NAPL saturated medium, forcing NAPL out of the interstices. The result is that sufficient head of NAPL must form on the surface before it displaces the water in the water saturated pores. After a spill occurs, a water-saturated soil may act to oppose NAPL infiltration, resulting in the spill pooling on the surface. In contrast, after a spill has occurred, rain may flush the NAPL from the near surface due to the NAPL being displaced by water, the wetting fluid.

In Figure 2.1, a capillary pressure exists between NAPL $\left(\mathrm{P}_{\mathrm{NAPL}}\right)$ and water $\left(\mathrm{P}_{\text {water }}\right)$, and between the air $\left(\mathrm{P}_{\text {air }}\right)$ and NAPL, as is the case in most three phase systems. The capillary pressure can be determined using the relation:

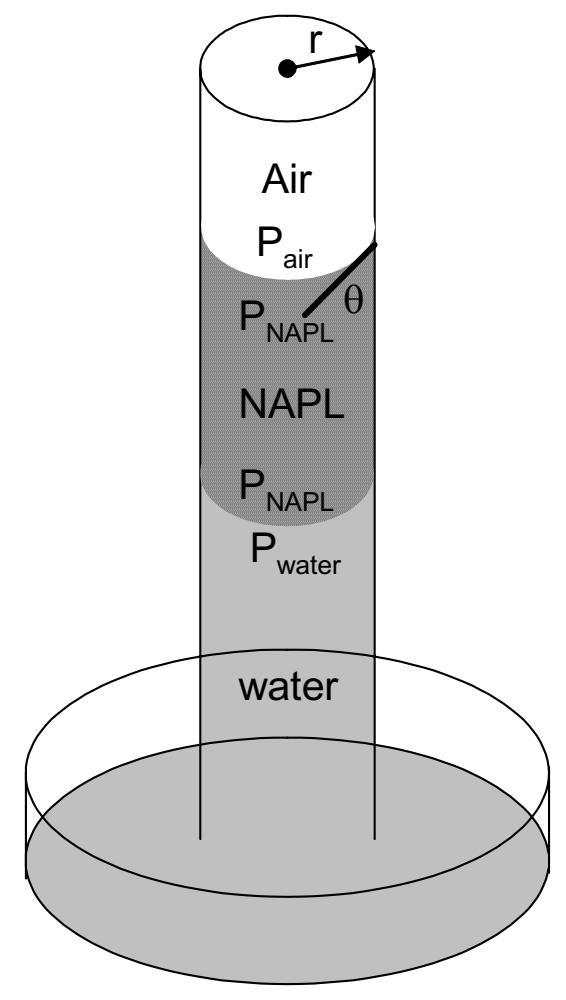

Figure 2.1. Capillary Tube Model 


$$
\Delta \mathrm{P}=\frac{2 \gamma \cos \theta}{\mathrm{r}}
$$

where $\Delta P=$ pressure difference between the wetting and non-wetting phase

$\gamma=$ surface tension

$\theta=$ contact angle (see Figure 2.1)

$r=$ capillary radius (see Figure 2.1)

Thus, capillary pressure is proportional to the surface tension and inversely related to the radius of the pore.

Because water in the column is being held up by a capillary force, we can view it as a suction force or negative pressure, holding the water column up against the downward pulling gravity and, in the case of Figure 2.1, the weight of the NAPL.

A retention relation is a functional connection between the liquid saturations and the capillary pressures. (Liquid saturation is the relative volume fraction occupied by a phase.) For a porous system with only water and air, there is a functional connection between the water saturation and the air-water capillary pressure. The fluid-media scaling principle based on ratios of interfacial tensions between the three fluids, air and NAPL, NAPL and water, in that wetting order, can be applied to produce the retention relations for the simultaneous retention of the three fluids. All that is required is the measured air-water retention relation.

Figure 2.2 displays air-water retention relations in three different media types: gravel, sand, and silt loam. Note that in a two-phase air and liquid system, the vertical equilibrium distribution of liquid held up against gravity is a direct indication of the retention relation, with the pressure expressed as the liquid height above the fully saturated level. Figure 2.2 can be explained by the capillary tube model because the average pore radius in a gravel soil would be larger then that found in sand, with the same being true of sand and silt loam. Because capillary pressure is inversely related to pore radius (Equation 2.4), a smaller $\Delta \mathrm{P}$ or suction would be needed to mobilize the water (decrease the water content) filling a gravel pore than would be needed in a sand or silt loam pore. Thus Figure 2.2 gives the relation of how tightly water is held in the soil and, as such, how easily it will infiltrate and drain. For example, the water content of the gravel begins to decrease at a lower suction than the sand and silt loam, representing less energy necessary for infiltration and drainage of the water to occur. An air-NAPL retention relation would display comparable behavior as that in Figure 2.2, with NAPL now being the wetting fluid. A similar relation between relative permeability and suction can be developed.

The relative permeability and capillary pressure relations determine constraints or connections between the saturations of each fluid present in a porous medium. These connections determine the coupling of the three fluid movement equations based on Darcy's law for each fluid. 


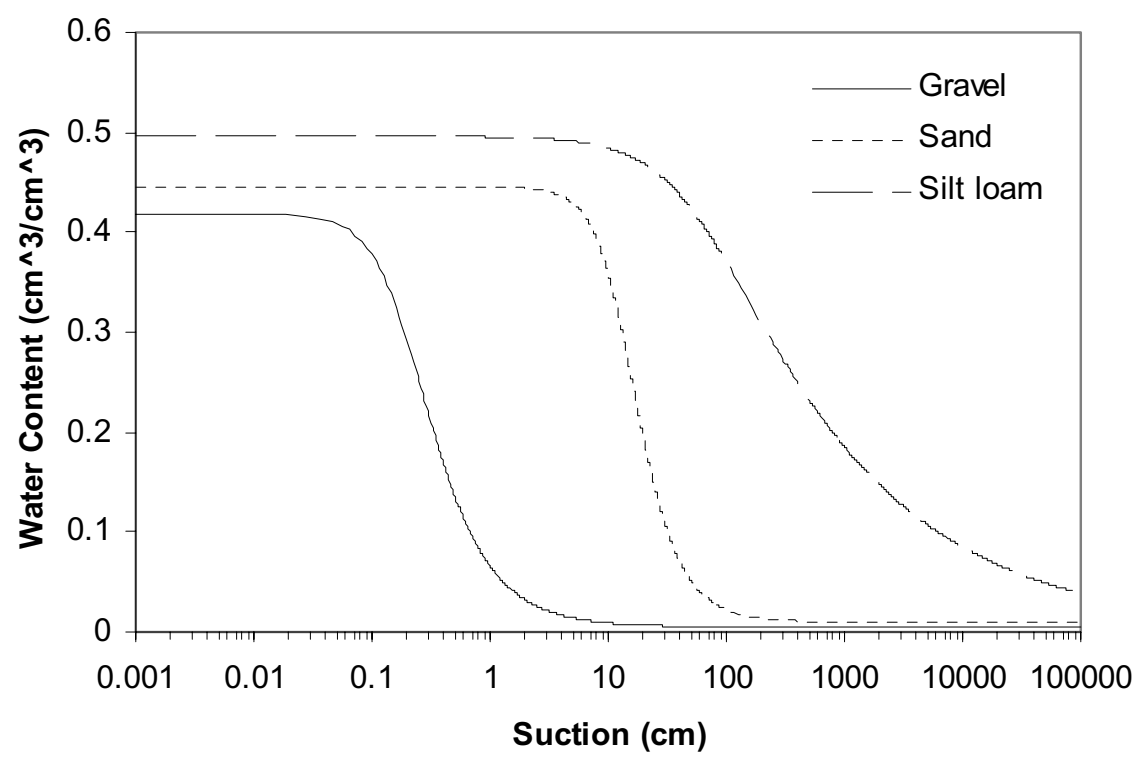

Figure 2.2. Air-Water Retention Relations for a Gravel, Sand, and Silt Loam

\subsubsection{Infiltration}

If NAPL is spilled onto the land surface, it experiences a pressure gradient downward due to its weight, which depends on the height of the liquid standing above the surface. However, it is also drawn into the subsurface medium by capillary suction or a capillary pressure gradient. Liquid conductivity as determined by the increasing liquid content determines how rapidly the infiltration front can move downward into the subsurface. The multiphase equations of fluid movement describe how quickly the infiltration would occur. A multiphase fluid flow equation solver is needed to calculate infiltration rate based on Darcy's law combined with the fluid constitutive theory, which is made up from the permeability functions and retention relations.

Parker (1989) gives an explanation of multiphase flow physics, which is more precise than given here. He explains how the flow of each fluid is mathematically connected in a porous medium. That reference summarizes the relevant equations and describes the fluid-media scaling principle. The fluid equations of motion are put together in a multiphase flow simulator described by Kaluarachchi and Parker (1989). A computer code is available to apply the theory for infiltration and drainage processes.

Another advanced multiphase flow simulator, having a different mathematical implementation, was described more recently by White, Oostrom, and Lenhard (1995). This simulator and solver of the multiphase flow equations are implemented in the STOMP code, which is available to this spill modeling effort.

The infiltration of NAPL into a water-unsaturated subsurface, called a vadose zone, can be simplified in various ways by imposing special circumstances. For instance, the movement of the gas (air) phase could be neglected if volatilization does not contribute substantially to the fluid composition or if the air 
phase can escape freely. This reduces the coupled motion equations to two, instead of three, and facilitates the computational burden of calculating NAPL infiltration.

\subsubsection{Simplified Infiltration Model}

An approximate equation for infiltration of water can be generalized to apply for a NAPL infiltrating into a partly water-wetted medium. However, when using this infiltration formula, the accuracy is unknown, until it is compared to exact theory. Reible et al. (1990) describe a simplified calculation to represent infiltration in a one-dimensional vertical case. Experimental measurements were performed to justify the approximation. Another similar model is described by Weaver et al. (1994). These are sharpfront models that neglect the dynamic complexity of NAPL entering a medium initially partly wetted by water. The infiltration front is described as a plug of NAPL moving downward under the pressure head of the applied (spilled) liquid. The movement or redistribution of the initial water, which would happen as a result of the change in interfacial capillary forces as NAPL infiltrates, is neglected. This may or may not be a reasonable approximation depending on many circumstances. The concept of the simplified infiltration model is demonstrated in a later section with an example.

\subsection{Spreading on the Surface}

There is substantial theory available for the movement of water over the land surface. However, those mathematical formulations have not been extended to account for a NAPL, as has the theory of multiphase flow in the subsurface. Fortunately, the modification needed to account for NAPL in overland flow only involves considering the different density and viscosity of a NAPL.

\subsubsection{Overland Flow}

A flow of liquid passing over a solid surface, either level or inclined, and under a less dense fluid (air in this case) is called a "gravity current" in fluid dynamics. Only recently have fluid dynamics researchers turned their attention to gravity currents on a deep porous surface. Acton et al. (2001) described a situation that could be used as a paradigm for a spill. They described a mathematical formulation and simple confirming experiments of a single viscous liquid flowing over an unlimited deep porous medium. Those authors point out that viscous flow over a permeable boundary has as yet received very little research attention. The fact that Hussein et al. (2002), who produced an even more recent publication, could not identify other models with similar purpose tends to reinforce the finding that such models are presently not available in the open literature.

The overland flow of water as a fluid dynamical process has concurrently been an important research topic with surface water hydrology and is typically described with the use of the Saint Venant equations (see Woolhiser and Liggett 1967). Although, it is only recently that surface water hydrologists have attempted to couple overland flow with surface infiltration at a spatial scale that is relevant to a spill. For instance, Esteves et al. (2000) claim that their approach to couple overland flow and infiltration modeling for small regions is original at the time. These theoretical models all include the basic elements of a liquid spill on land, but none treat the general complexity of a NAPL undergoing three-phase infiltration as coupled to its overland flow over a tilted topography. 
Zhang and Cundy (1989) describe a mathematical formulation for overland water flow, which is potentially adaptable for NAPL. Fluid dynamic equations are developed that account for surface topography, roughness of the surface, and the loss of water through infiltration. Viscous shear stress to account for surface roughness is described in the model. The mathematics for the fluid dynamics is appropriately chosen to deal with smaller sloping regions, which might be more appropriate for the spatial scale of a spill. The fluid dynamic equations obtained represent shallow flow with the drag on the downhill movement being accounted for by the shear stress.

Esteves et al. (2000) took the same shallow overland flow model and included a simple description of infiltration. They use the simplified Green-Ampt model to describe infiltration. Esteves et al. (2000) point out that the model is based on the Saint Venant equations, commonly employed in modeling runoff from land surfaces. These equations of shallow overland flow account for slope variation and friction drag on the liquid. Liquid flows down slope and disappears at locations where the infiltration capacity exceeds the rate of overland supply. If the arrival of liquid is larger than infiltration capacity, the head or height of liquid builds up as it continues down the slope.

The infiltration loss of liquid may not always result from a uniformly porous subsurface medium. Flow into a distribution of macropores or fissures might increase the infiltration during overland movement of a spill. Leonard et al. (2001) have discussed another adaptation of the same surface flow model to include macroporous infiltration.

These models, cited above, require solving a complicated set of equations that tend to have numerical instability difficulty. Thus obtaining an accurate solution is not an easy task when this approach for describing surface flow is taken. However, Fennema and Chaudhry (1990) give one of many available mathematical studies of how the Saint Venant equations can be solved.

\subsubsection{Simplified Spreading Model}

Hussein et al. (2002) developed a spill spreading model that avoids the complication of overland flow by using concepts from lubrication theory. They describe the overland flow as a gravity current on either a simple flat region or a single sloping plane. The screening-spreading model is devised by coupling infiltration as described by the Green-Ampt equation with a gravity current equation. The mathematical theory for gravity currents is considerably developed and provides an alternative viewpoint for overland flow.

Whereas the model of Hussein et al. (2002) makes use of an approximate solution to the problem of liquid spreading with loss by infiltration, Acton et al. (2001) have formulated an exact coupling of the two processes. However, the model's applicability to real soil conditions is limited because the downward percolation of liquid is formulated as saturated flow. Nevertheless, the model would be useful to gain a quantitative picture of the detailed balance between how fast and far a liquid would spread in relation to the magnitude of the porous permeability. The similarity solutions obtainable for gravity currents also provide insights into how far spreading takes place depending on how rapidly the spill is released. 


\subsection{Spill Disappearance}

Once a spill has occurred and spread to its maximum extent, the NAPL wetted region on the surface is all that remains visible. For certain subsurface soils, the medium stays darker in NAPL wetted spots. How quickly the NAPL drains from the surface after entering the subsurface is going to control how long it remains detectable. Evaporation can also reduce the surface liquid content if the NAPL is volatile. However, evaporation can also contribute to maintaining the liquid content at the surface by producing a surface withdrawal that is re-supplied by unsaturated conduction of the NAPL from its storage in the subsurface.

Generally, multiphase flow theory is applied given specified initial and boundary conditions to determine the subsurface dispersal of NAPL contaminants. Most subsurface modeling was devised to examine the subsurface fate of contaminants given the surface behavior as a known boundary condition. The spill problem, however, calls for the reverse of determining the surface fate of a NAPL, while remaining detectable. Aside from models that assume that a NAPL source remains on the surface while it evaporates or weathers, there does not seem to be any models that deal directly with the fate of surface NAPL. This deficiency, however, might be overcome if existing theory for the behavior of water in unsaturated media at the land surface can be modified to describe a nonaqueous phase as well as water.

Another fundamental problem with estimating the disappearance of NAPL within the land surface is that an arbitrarily shallow depth segment of surface material usually does not reflect the physical properties of the greater volume of subsurface media that might be characterized or catalogued. That is, the first centimeter or so of subsurface is usually quite distinct physically from deeper geologic media, because it has been subject to climatic or human alteration over time. For instance, even if the surface soil is the same geochemical composition as the subsurface, the grain size distribution and packing are usually entirely distinct. In addition, considerable biological or microbial material may be present on the surface. Thus the first centimeter of surface is likely to constitute a unique porous system coupled only weakly to the NAPL flow behavior occurring within the deeper subsurface.

This situation seems to call for an entirely different pore-scale viewpoint on the potential fate of a spilled NAPL remaining near the surface. Fortunately, many of the same physical/chemical phenomena apply to the surface porous media, but the challenge would be to obtain its specific NAPL retention properties.

A pore-scale perspective, as might be needed to address the surface behavior of NAPL, may be found in the discussion by Gvirtzman and Roberts (1991). They discuss how two immiscible fluids conform to ideal media particles (packed glass beads). The NAPL retention behavior can then be connected with entirely fundamental physical parameters. Kao and Hunt (1996) further reinforce this concept that a capillary pore-scale viewpoint can be employed to explain NAPL movement, requiring only a minimum of phenomenological parameters. McBride et al. (1992) also showed how the relative absorption behavior of NAPL can be compared using a simple capillary model. Using these sorts of simplified conceptual concepts of NAPL retention and movement, it may be possible to construct a very mathematically simple description of the amount of NAPL present over time following a spill. In such a model, the particular surface distribution of a spill and the exact total quantities released into the ground are not crucial or necessary. By focusing on only the surface layer of the land, with rates of NAPL disappearance 
determined by atmospheric-driven evaporation and drainage loss to the deep subsurface, it should be possible to construct an appropriate model gauging the detection of NAPL by infrared spectroscopy or any other surface measuring system.

\subsubsection{Drainage of NAPL}

A spilled NAPL would be coupled to the surface by the behavior of gravity drainage in the subsurface. As drainage occurs from below, surface NAPL concentrations would be drawn down by capillary action. The distribution of a NAPL with depth would follow at a rate determined by the intrinsic permeability and viscosity and driven by gravity. The distribution would eventually move toward a balance determined by capillary retention while approaching equilibrium with the subsurface storage of the spill. In principle, the multiphase flow theory could predict this behavior, provided the surface and subsurface material properties are accurately known, but this information is often time consuming and costly to obtain. At the same time that drainage is taking place, the NAPL may be evaporating from the surface, so that two fluxes, one driven by the subsurface and the other by the atmospheric boundary, are working in conjunction to diminish the surface NAPL content.

No models were found that deal directly with an emphasis on the behavior of NAPL in the first centimeter of the surface. Using fluid-media scaling, it may be possible to develop a simplified screening description. For instance, an analytical description of soil water drainage in an unsaturated profile could be modified to describe NAPL rather than water. Warrick et al. (1990) derived an analytical solution to the Richards unsaturated flow equation for some special representations of a porous medium. The work was later generalized by Warrick et al. (1991) for a time-varying flux imposed at the surface. These solutions could be modified for a different density, interfacial tension, and viscosity to describe a particular NAPL. Being analytical, although not an absolutely accurate representation of every soil medium, this solution of the basic porous flow equation could provide an easily implemented calculation, free of the complexity and difficulty of using a full multiphase numerical simulator.

\subsection{Evaporation Loss from the Surface}

Evaporation of a liquid residing on a surface has been extensively studied, largely due to the interest in the fate of oceanic spills of crude oil and to a lesser extent the fate of applied pesticides. Depending on the chemical properties of the spill, properties of the soil, and environmental conditions, a surface spill may experience significant evaporative loss. Oceanic oil spills have undergone a 30 percent to 60 percent reduction of spill mass purely due to evaporation (NAS 2003). Additionally, up to 50 percent of a pesticide application may be lost due to volatilization (Van Den Berg et al. 1999).

\subsubsection{Evaporation}

Evaporation of a liquid is determined largely by the liquid's vapor pressure (Schwarzenbach et al. 1993). Simply stated, vapor pressure is the pressure exerted by gas molecules of a compound in equilibrium contact with its condensed (liquid) phase. Thus the larger a compound's vapor pressure, the greater its affinity for evaporation. In terms of a pure liquid, knowledge of a compound's vapor pressure allows evaporation of that compound to be described by a molecular diffusion process of the form: 


$$
\text { Evaporative Flux }(\mathrm{N})=\mathrm{K}_{\mathrm{m}} \frac{\left(\mathrm{P}_{\mathrm{s}}-\mathrm{P}_{\infty}\right)}{\mathrm{RT}_{1}}
$$

where $K_{m}=$ gas-phase mass transfer coefficient

$$
\begin{aligned}
R= & \text { gas constant } \\
T_{l}= & \text { temperature of the liquid } \\
P_{s}= & \text { compound vapor pressure at the surface of the spill } \\
P_{\infty}= & \text { vapor pressure on the atmosphere side of the boundary layer or at infinite altitude of the } \\
& \text { atmosphere, which for simplicity is usually taken to equal zero in either case (Mackay and } \\
& \text { Matsugu 1973; Jury et al. 1983). }
\end{aligned}
$$

The gas-phase mass transfer coefficient accounts for additional factors that effect evaporation, most notably being wind shear stress at the liquid surface, usually correlated to wind speed, spill surface area, chemical diffusivity in air, and stagnant air boundary layer thickness. Numerous relationships have been developed to determine the gas-phase mass transfer coefficient, all requiring the determination of one or more compound-specific constants. A description of the more established relationships can be found in Fingas (1995) and Yates et al. (2002). Because the constants making up the relationships are compound specific, multiple gas-phase mass transfer coefficients may be needed to fully describe volatilization of a multi-component liquid.

A compound's vapor pressure has the potential to change by more than an order of magnitude over the ambient temperature range (Schwarzenbach et al. 1993). Thus, the effect of temperature on evaporation is inherent in Equation (2.5) and requires a good working knowledge of the compounds temperaturevapor pressure relationship. This relationship can be attained for a variety of compounds using the Antoine equation and information in the CRC Handbook of Chemistry and Physics (Lide 2002).

The vapor pressure of a multi-component liquid is not only dependent on liquid temperature but also on the individual component vapor pressures and the fraction of each component making up the liquid. Researchers have observed that a multi-component liquid experiences an exponential loss of the more volatile components with time (ASCE 1996). The loss of the more volatile components results in the vapor pressure decreasing with time. Thus, as evaporation progresses, an accounting of the pool's composition is needed to accurately describe evaporation of a multi-component liquid.

Once infiltration of the spilled liquid has occurred, the liquid may still experience a significant evaporative flux directed towards the soil surface. This requires the consideration of two additional processes, mass transfer between the infiltrated liquid and the gas within the soil matrix and advectiondiffusion of the gas to the soil-atmosphere boundary where it may diffuse out of the soil as described by Equation (2.5). Additional subsurface processes such as sorption to subsurface materials, degradation, and heat movement may influence the gas flux towards the soil surface (Jury et al. 1983; Parlange et al. 1998; Yates et al. 2002). Mercer and Cohen (1990) provide a detailed description of the volatilization and transport of chemicals in the soil gas. 


\subsection{Spill Chemical Weathering}

Chemical weathering pertains to the chemical, photochemical, or biological mediated transformation of a substance into a chemically different entity. Compared to spill infiltration and evaporation, pronounced chemical weathering may not occur until long after the spill has taken place. As a result, consideration of chemical weathering will be of most importance with low volatile spills that remain on or near the surface. Due to the complexity and interrelation of chemical reactions, it may not always be obvious which process (chemical, photochemical, biological) or group of processes contributed to chemical weathering, complicating analysis. Important considerations when investigating chemical weathering are what the reaction products are, what the kinetics or overall rate of the reaction is, and what environmental variables influence the reaction, such as temperature, $\mathrm{pH}$, redox condition, ionic strength, and solute concentration. Schwarzenbach et al. (1993) provide a thorough discussion on the theory and quantifycation of all three weathering processes.

\subsubsection{Chemical Transformation}

Chemical transformations account for reactions that can occur without the assistance of microorganisms. Chemical reactions are often described by a collision rate model, which usually states that a reaction can occur only if reactants encounter each other by colliding with sufficient kinetic energy to break relevant bonds. Based on such models, the rate of a reaction may be considered a function of the frequency of reactant encounters, which is proportional to concentration, and their kinetic energy, which is related to temperature. Thus spilled NAPL on the ground is subject to chemical breakdown caused by prevailing temperature and climate. The transformation rate is often described mathematically by the first-order rate law:

$$
[\mathrm{A}]=[\mathrm{A}]_{\mathrm{o}} \mathrm{e}^{-\mathrm{kt}}
$$

where $[\mathrm{A}]_{\mathrm{o}}=$ the initial concentration of the reactant

$[\mathrm{A}]=$ the concentration of the reactant at some time $\mathrm{t}$

$\mathrm{k}=$ reaction rate constant.

\subsubsection{Photochemical Transformation}

Photochemical transformation describes the transformation of a chemical species due to its increased reactivity as a result of the absorption of light or by reaction with chemical species that have become highly reactive due to incidence of sunlight. The rate of photochemical transformation is a function of both the light absorption spectrum of the compound and environmental factors that affect the solar spectrum, such as soil type, soil layer thickness, latitude and longitude, season, time of day, and weather conditions. 


\subsubsection{Microbial Transformation}

Microbial transformation, or biodegradation, is the breakdown of chemical species due to microbial metabolism. Biodegradation is a critical chemical weathering process because in many instances it is the only process by which an organic compound may be mineralized in the environment. The most important factors influencing microbial activity and their transformation of chemical species are oxygen availability, organic matter content, nitrogen availability, and contaminant bioavailability. Although anaerobic biodegradation is recognized as an important degradation pathway, it is commonly much slower than aerobic biodegradation. The abundance of carbon in the form of organic matter is important for cell maintenance and growth. While most surface soils generally contain a sufficient amount of organic matter to sustain large numbers of microorganisms, this may not be true of soils in dry regions which support very little flora. The consequence of low organic matter content is that once a substrate, such as a spill, is added to the soil, the period of time until the microbial population reaches a viable size may be prolonged. Once a spill does occur, the sorption behavior and solubility of the chemical species may limit the availability of that species to the microorganism. A thorough review of the phenomena that control biodegradation is presented by Sturman et al. (1995).

Monod kinetics is often used to describe microbial population growth and subsequently the rate of microbial transformation. In Monod kinetics the substrate of concern (spilled liquid) is considered nonlimiting in terms of cellular growth. Under this condition, once the substrate is added, microbial population growth increases exponentially until some limiting factor restricts continued population growth. Relating the creation of cellular biomass to transformation of a chemical species allows the microbial transformation rate to be established.

In summary, theories of phenomena presented in the previous sections become extremely involved when dealing with three-phase (water-gas-NAPL) systems, which occur in NAPL spill scenarios. The complexity increases even more when the spilled liquid itself is composed of multiple components. Then certain assumptions may be made to simplify the problem, but this may come at the expense of model accuracy. Thus a balance must be achieved between computational efficiency and model definitiveness. Section 3 discusses parameters, which are incorporated into the described theories to represent all or partial aspects of a spill to varying degrees of complexity. Section 4 demonstrates the use of some of the basic parameters along with the spill modeling concepts previously discussed. 


\subsection{Parameter Needs for Spill Modeling}

A variety of spill models identifying exactly what input parameters are required was not found. Nevertheless, it is possible to identify the essential parameters that are likely needed in any model's formulation for describing the spill behavior of a NAPL. In this section, the basic physical and chemical parameters or material attributes are identified along with a description of the likely role they play.

Table 3.1 contains likely parameters or attributes required to quantify spill behavior. The table also identifies available parameter sources along with the common units and symbols used to describe the parameters.

Many of the parameter values listed are affected by the properties of water in the earth. For example, $\mathrm{pH}$ and salt concentration will affect surface tension and solubility, to name a few. Especially important is that many of these parameters depend greatly on the temperature of the environment they happen to enter. Their values and usage will depend on the temperature.

\subsection{Fluid Properties}

\subsubsection{Density of NAPL}

Density is simply the mass of the liquid divided by the volume it occupies under the prevailing thermodynamic conditions of pressure and temperature. The density of a NAPL relative to water is of

Table 3.1. Spill Model Fluid Parameters, Units, Symbols, and Sources of Information

\begin{tabular}{|c|c|c|c|}
\hline Parameters & Units & Symbol & Information Source \\
\hline Density & $\mathrm{kg} \cdot \mathrm{m}^{-3}, \mathrm{~g} \cdot \mathrm{cm}^{-3}, \mathrm{lb} \cdot \mathrm{ft}^{-3}$ & $\rho$ & DIPPR $^{(a)}$ \\
\hline Viscosity & $\mathrm{kg} \cdot \mathrm{m}^{-1} \cdot \mathrm{s}^{-1}$ (centipose), $\mathrm{N} \cdot \mathrm{s} \cdot \mathrm{m}^{-2}, \mathrm{lb}-\mathrm{s} \cdot \mathrm{ft}^{-2}$ & $\eta, \mu$ & DIPPR \\
\hline Surface Tension & $\mathrm{N} \cdot \mathrm{m}^{-1}, \mathrm{~kg} \cdot \mathrm{s}^{-2}$, dyn $\cdot \mathrm{cm}^{-1}$, erg $\cdot \mathrm{cm}^{-2}, \mathrm{~J} \cdot \mathrm{m}^{-2}$ & $\sigma, \gamma$ & Mercer and Cohen (1990), DIPPR \\
\hline Interfacial Tension & $\mathrm{N} \cdot \mathrm{m}^{-1}, \mathrm{~kg} \cdot \mathrm{s}^{-2}$, dyn $\cdot \mathrm{cm}^{-1}, \mathrm{erg} \cdot \mathrm{cm}^{-2}, \mathrm{~J} \cdot \mathrm{m}^{-2}$ & $\sigma$ & Mercer and Cohen (1990) \\
\hline Wettability & radians, degrees & $\phi, \mathrm{rad}$ & Mercer and Cohen (1990) \\
\hline Vapor Pressure & $\begin{array}{l}\mathrm{N} \cdot \mathrm{m}^{-2}, \mathrm{~kg} \cdot \mathrm{m}^{-1} \cdot \mathrm{s}^{-2}, \text { pascal }(\mathrm{Pa}), \mathrm{bar}, \text { atmosphere }(\mathrm{atm}), \\
\text { torr, millimeter mercury }(\mathrm{mm} \mathrm{Hg}), \mathrm{lb}^{-2} \mathrm{in}^{-2}(\mathrm{psi})\end{array}$ & $\mathrm{P}, \rho$ & $\begin{array}{l}\text { Mercer and Cohen (1990), } \\
\text { Schwarzenbach et al. (1993), } \\
\text { DIPPR, PNNL IR Spectral Library }\end{array}$ \\
\hline Phase Equation & - & - & DIPPR \\
\hline Solubility & $\begin{array}{l}\text { Mol } \cdot \mathrm{L}^{-1}\left(\text { molarity), mol } \cdot \mathrm{kg}^{-1} \text { (molality), } \mathrm{mg} \cdot \mathrm{L}^{-1}\right. \\
(\mathrm{ppm}), \mathrm{mg} \cdot \mathrm{kg}^{-1}(\mathrm{ppm}), \mu \mathrm{g} \cdot \mathrm{L}^{-1}(\mathrm{ppb}), \mu \mathrm{g} \cdot \mathrm{kg}^{-1}(\mathrm{ppb})\end{array}$ & $\mathrm{C}^{\mathrm{sat}}$ & $\begin{array}{l}\text { Mercer and Cohen (1990), } \\
\text { Schwarzenbach et al. (1993) }\end{array}$ \\
\hline Residual Saturation & $\mathrm{m}^{3} \cdot \mathrm{m}^{-3}, \mathrm{~cm}^{3} \cdot \mathrm{cm}^{-3}$ & $\mathrm{~S}_{\mathrm{r}}$ & $\begin{array}{l}\text { Mercer and Cohen (1990), } \\
\text { Dombrowski and Brownell (1954) }\end{array}$ \\
\hline Air Diffusivity & $\mathrm{m}^{2} \cdot \mathrm{s}^{-1}, \mathrm{~cm}^{2} \cdot \mathrm{s}^{-1}, \mathrm{ft}^{2} \cdot \mathrm{s}^{-1}$ & $\mathrm{D}$ & $\begin{array}{l}\text { Schwarzenbach et al. (1993), Hillel } \\
\text { (1998) }\end{array}$ \\
\hline Henry's Constant & $\mathrm{atm} \cdot \mathrm{L} \cdot \mathrm{mol}^{-1}, \mathrm{~mol}^{-1} \mathrm{~L}^{-1} \cdot \mathrm{mol}^{-1} \cdot \mathrm{L}$ & $\mathrm{K}_{\mathrm{H}}$ & $\begin{array}{l}\text { Mercer and Cohen (1990), } \\
\text { Schwarzenbach et al. (1993) }\end{array}$ \\
\hline $\begin{array}{l}\text { First Order Degradation } \\
\text { Coefficient }\end{array}$ & $\mathrm{s}^{-1}$ & $\mu$ & - \\
\hline
\end{tabular}


major importance. Some NAPLs are denser than water and will sink into groundwater, especially if the water table is near the land surface. Density determines the inertia effects, with gravitational forces being greater on fluid with greater density.

\subsubsection{Viscosity}

Viscosity quantifies the internal friction of a liquid and determines how rapidly a liquid flows over a surface as a result of gravity acting on the liquid. Viscosity is required to calculate the liquid conductivity along with intrinsic permeability and density. If a NAPL is highly soluble in water, the fluid viscosity of water may be substantially altered by the amount of NAPL dissolved. Viscosity is a thermodynamically dependent parameter, and whether the earth surface is relatively hot or cold determines how fast a spill may spread. In conjunction with permeability, viscosity also determines how rapidly the liquid might be absorbed into the porous subsurface. More viscous liquids will flow more slowly overland and infiltrate less rapidly than less viscous liquids. Water may be used as the reference liquid for comparison with NAPLs.

\subsubsection{Surface Tension}

Surface tension is the potential energy stored in the surface configuration of a liquid when spread. In addition, surface tension can be described as the mechanical work done per unit area in creating a liquid interface in contact with air. Liquids with greater surface tension require greater force to stretch. Greater surface tension is associated with greater capillary rise height, assuming two compared liquids have the same wetting character or contact angle. Surface tension can be thought of as the force per unit length taken along the wetting contact line of a liquid with the solid it is adherent to. Mercer and Cohen (1990) describe in detail the relationship of surface tension and interfacial tension to the behavior of a NAPL in a porous medium.

\subsubsection{Interfacial Tension}

Interfacial tension is the potential energy associated with the area of the contact between two liquids, NAPL and water. Consider a layer of NAPL over a container of water in which the NAPL is less dense than water and immiscible, as is commonly true of hydrocarbons. Next, consider a glass capillary tube placed vertically through the NAPL layer floating over the water interface. Generally, water is more wetting on glass or silicon substrate than most NAPLs. That is, water adheres to glass or sand grain surfaces more strongly than most NAPLs. The water will rise to a certain height in the tube (if sufficiently narrow to detect the capillarity) and displace a volume of NAPL originally filling the tube above the starting interface (Figure 2.1). The capillary rise height is directly proportional to the product of interfacial tension and the cosine of the contact angle with the glass tube wall. The shape of the interface in the capillary tube is concave downward compared with the flat surface between the liquids outside the tube. The angle that the interface surface (the tangent to the surface) makes with the glass wall is the contact angle. Note that the contact curve (a circle) where the NAPL-water interface meets the glass wall is above the bottom concavity of the interfacial surface. This is because the water preferentially wets the glass. The greatest water wettability is reflected by a contact angle of zero. This is when the liquid interface is exactly tangent to the tube's wall where it contacts. Greater contact angles up to 90 degrees indicate less strong adhesion of water compared to the NAPL. In addition, the rise height is 
inversely proportional to the product of the tube radius with the density difference between water and NAPL. The smaller the density difference, the greater the rise height, and the height is greater for a smaller tube radius. One can also visualize interfacial tension in terms of a drop of NAPL (less dense) placed on a water surface held in a dish.

\subsubsection{Wettability}

Wettability was introduced with the interfacial tension. If water is the wetting fluid, then the contact angle of the water-NAPL interface in a glass capillary tube is a value between zero and 90 degrees. For example, consider a drop of water sunk to the bottom of a dish of NAPL. The drop of water is spread out along the bottom of a glass dish if it wets more strongly than NAPL. In the opposite case, when a NAPL is more wetting (say for a plastic dish), the water drop forms a bead not spreading against the dish. Mercer and Cohen (1990) describe this property with figures. Cosine of the contact angle is related to the three interfacial tensions between the liquids and air and the interfacial tension between the NAPL and water. That cosine of the contact angle quantifies the wettability.

\subsubsection{Vapor Pressure}

Pressure is exerted by all molecules of evaporated liquid held in a sealed container having air space above the NAPL surface. Both water and NAPL have a vapor pressure, and each contribute a partial pressure. NAPLs with greater vapor pressure are usually more volatile and evaporate more rapidly into the air over a spill. Vapor pressure is greater for greater temperature. Physical models for NAPL evaporation depend on vapor pressure differences going from the porous medium into the atmosphere above.

\subsubsection{Phase Equation}

Many of the properties of a NAPL are given by its thermodynamic behavior. A phase equation indicates if the substance is liquid or gas under conditions of given pressure and temperature. Information needed to construct a phase equation includes critical temperature, pressure, volume, and the triple point.

\subsubsection{Solubility}

Solubility indicates how much NAPL will dissolve in a certain amount of water. If a NAPL is highly soluble, it could enter the subsurface or go below the groundwater table by dissolving directly into the water, rather than displacing the water by capillarity. Solubility also affects the interfacial tension between water and NAPL.

\subsubsection{Residual Saturation}

If a spill of NAPL into the porous subsurface is flushed out by rainfall or overland runoff, for example, a certain volume of NAPL may remain. Measurements of residual saturations, if available, might be directly useful to estimate how much spilled liquid content could be present following rainfall. The problem of detecting and evaluating a spilled NAPL might be considerably simplified by considering only the likely residual that would remain near the surface to be measured by infrared scanning. 


\subsubsection{Air Diffusivity}

Air diffusivity reflects the random movement of molecules through air by Brownian motion. Air diffusivity is inversely related to molecular size due to the decrease in mean Brownian motion velocity and mean free path length as molecular size increases. When quantifying solute movement, air diffusivity is an important parameter particularly when advective transport is minimal. It will be necessary to determine an effective air diffusivity when quantifying diffusive solute movement through the soil air fraction. The use of an effective diffusivity is necessary due to the limitation of continuous air-filled pores and the tortuous nature of the pores. Many approaches used to determine air diffusivity involve the use of the chemical's molecular mass as a measure of the molecular size, which is then related to air diffusivity. Relationships between effective air diffusivity and soil air fraction have been developed by a number of researchers, as described by Hillel (1998).

\subsubsection{Henry's Constant}

Henry's constant describes a compound's air-water distribution ratio at equilibrium, representing that compound's liquid-gas partitioning behavior. Determining the liquid-gas phase partitioning behavior of a solute will aid in determining the infiltrated spill fraction that is present as a gas, where it may then experience gas-phase diffusive-advective transport towards the soil-atmosphere interface.

\subsubsection{Degradation Coefficient}

A degradation coefficient relates the amount of chemical lost with time due to chemical weathering processes. Because it is difficult to determine to what extent each separate chemical weathering process degrades a chemical, a single degradation coefficient is used. Large variability in degradation coefficients exists for a single chemical species due to variability in degradation factors such as temperature and microbial population. As such, degradation coefficients are difficult to accurately assess and apply.

\subsection{Subsurface Media Properties}

This section describes the subsurface media properties used in spill models. The parameters are listed in Table 3.2.

\subsubsection{Soil Type}

The size and interconnectedness of the interstices (pores) of a subsurface medium control how rapidly a spilled liquid infiltrates. The pore space reflects, to a considerable extent, the size of the soil grains composing the porous medium. Generally, soils are classified by fractions of sand, silt, and clay. Each of these soil categories represents a certain grain size distribution. The importance of soil type is that it is related to the liquid retention and conduction behavior. Very often soil type can be correlated with more specific (mathematical) descriptions of the soil water retention curve. Marshall and Holmes (1979) in the first chapter of their text give a brief discussion of soil type. Soil type can also represent the geochemical (mineral) composition of the grains. Chemical composition can have a great deal of influence on how the liquid interacts with the medium, especially for infrared detection of a chemical's signature. In a sand, 
Table 3.2. Spill Model Subsurface Media Parameters, Units, Symbols, and Sources of Information

\begin{tabular}{|c|c|c|c|}
\hline Parameter & Unit & Symbol & Information Source \\
\hline Soil Type & $\begin{array}{l}\text { sand }(2 \mathrm{~mm}-0.05 \mathrm{~mm}), \text { silt }(0.05 \mathrm{~mm}-0.002 \mathrm{~mm}) \text { clay }(< \\
0.002 \mathrm{~mm})^{(\mathrm{a})}\end{array}$ & - & NRCS soil survey \\
\hline Mineral Content & - & - & - \\
\hline Bulk Density & $\mathrm{kg} \cdot \mathrm{m}^{-3}, \mathrm{~g} \cdot \mathrm{cm}^{-3}, \mathrm{lb} \cdot \mathrm{ft}^{-3}$ & $\rho_{\mathrm{b}}, \mathrm{D}_{\mathrm{b}}$ & NRCS soil survey \\
\hline Porosity & $\mathrm{m}^{3} \cdot \mathrm{m}^{-3}, \mathrm{~cm}^{3} \cdot \mathrm{cm}^{-3}$ & $f, n, \phi, \varphi$ & - \\
\hline $\begin{array}{l}\text { Soil Water Retention } \\
\text { Curve }\end{array}$ & $\begin{array}{l}\text { Erg } \cdot g^{-1} \text { vs. water content, joule } \cdot \mathrm{kg}^{-1} \text { vs. water content, bar vs. } \\
\text { water content, } \mathrm{cm} \mathrm{H}_{2} \mathrm{O} \text { vs. water content }\end{array}$ & - & $\begin{array}{l}\text { UNSODA, }^{(b)} \\
\text { ROSETTA }^{(c)}\end{array}$ \\
\hline Intrinsic Permeability & $\mathrm{m}^{2}, \mathrm{~cm}^{2}, \mathrm{ft}^{2}$ & $\mathrm{k}$ & - \\
\hline Grain Size Distribution & - & - & - \\
\hline $\begin{array}{l}\text { Vapor Diffusion } \\
\text { Coefficient }\end{array}$ & $\mathrm{m}^{2} \cdot \mathrm{s}^{-1}, \mathrm{~cm}^{2} \cdot \mathrm{s}^{-1}, \mathrm{ft}^{2} \cdot \mathrm{s}^{-1}$ & $\mathrm{D}, \mathrm{D}_{\mathrm{s}}, \mathrm{D}_{\mathrm{v}}$ & - \\
\hline \multicolumn{4}{|c|}{$\begin{array}{l}\text { NRCS = Natural Resources Conservation Service. } \\
\text { (a) Numbers in parenthesis indicate particle diameter range from USDA classification scheme. } \\
\text { (b) Nemes et al. (2001). } \\
\text { (c) Schaap et al. (2001). }\end{array}$} \\
\hline
\end{tabular}

water is the wetting liquid, but in clay, the NAPL might be the wetting liquid. The wetting character determines which liquid in a water-NAPL system is held more strongly in the smaller interstices. Wettability depends on the soil type.

\subsubsection{Mineral Content}

The mineral makeup of the porous media is typically associated with the soil type (assuming an unconsolidated or granular surface medium). Sand is usually composed of quartz. Clay can be classified as made up of three basic kinds of minerals: kaolinite, illite, and montmorillonite. The water retention behavior of clays is chemically complicated, as is the interaction of various NAPL types with clay. Clays contain molecules of silicon dioxide in a crystal lattice layered structure. In clay, the retention of water is strongly controlled by chemical bonding, whereas in simpler granular sand, fluids are mainly held by physical capillarity. Most importantly, the majority of soils are composed of a combination of sands and clays aggregated in complicated grains. This variety of subsurface soils is the primary source of difficulty when predicting water retention behavior of soils. Marshall and Holmes (1979) discuss this subject in greater detail.

\subsubsection{Bulk Density}

Bulk density is the mass of the entire medium per unit volume as it is while in situ (solids and pores). The volume of a sample core and the collected mass would define this density, as opposed to the inherent grain density. Bulk density indicates the degree of physical packing of soil grains, the likely volume of interstices, and the pore size distribution associated with a particular soil sample. A basic problem with regards to classifying soils for spill situations is that the bulk density, and hence the fluid conduction properties, of a surface medium is usually different from the subsurface medium. Therefore, catalogues of soil types and their associated hydraulic properties will likely not represent the land surface very well. However, it may be possible using bulk density and grain size distribution data to estimate hydraulic 
properties for surface soils. Special theoretical models may be used to estimate hydraulic properties from bulk density and particle-size distributions (Arya and Paris 1981). There have been many advances and improvements to this basic hydraulic property model since its publication.

\subsubsection{Porosity}

Porosity is the volume of interstices per bulk volume of a porous medium, and thus the volume available to hold a water or NAPL. It can be measured directly as the most basic attribute of a porous medium, or it can be estimated from bulk density and particle density. Typically, in fluid flow modeling the liquid content divided by porosity is given as the relative saturation. The relative saturation variable is useful because it normalizes all fluid variation to the same scale between zero and unity.

\subsubsection{Soil Water Retention}

Soil water retention is the most basic of hydraulic functions describing how strongly water is retained by soil for specific water content. Water content is the volume of water held per unit volume of medium. The maximum water content can ideally be the porosity. However, that generally never occurs because air is invariably trapped in the pore space when water is imbibed. The scaling theory of multiphase flow provides a means for describing the three-phase retention of water, NAPL, and air in a porous medium starting with only the soil water retention curve.

Consider a column filled with a granular medium or a soil type with a retaining fine screen to keep the material from falling out. The granular material is packed in the column to a particular bulk density. Suppose the bottom of the filled column with the screen is just dipped in a water dish-the water level in the dish will be maintained at fixed level. The water will imbibe into the column moving upward. (We make the column sufficiently long by trial and error so that the water never reaches the top.) This same test works also using a NAPL instead. Finally (maybe after considerable time depending on the particular soil type), the amount of water imbibed or absorbed by the column will be constant. If after time, the column is sectioned and the water content measured at incremental height above the starting water level, the water content will decrease from a maximum saturated value to nearly nothing. The height above the starting reference represents potential energy of the water at the volumetric content as measured. Water adhesion to the matrix and capillarity draw the water in until it reaches an equilibrium with gravity. One can visualize the porous column as a size-distributed bundle of capillary tubes. Only the smallest radii tubes will conduct the water to the greater heights. In the column, there generally will be a saturated region of some height just above the starting water level. This is the so-called capillary fringe. Because water in the column is being held up by capillary force, against the downward pulling gravity, one can view it as a suction force or negative pressure. The rise height can be converted into the matric potential energy or defined as capillary pressure, which is the pressure difference between pressures in the air and liquid phases (i.e., non-wetting phase pressure minus the wetting phase pressure). Note that the suction pressure or capillary pressure is defined to be positive, but the matric potential energy or pressure head is negative. Finally, note that there are measuring instruments (tensiometer) that allow the suctions and fluid contents to be measured in situ simultaneously.

Again, Marshall and Holmes (1979), or nearly any other text on soil physics, is a source of information on this soil and water property. A fundamental assumption of unsaturated flow physics is 
that this retention relation applies even while the liquid is moving upward to the final static equilibrium distribution. Darcy's law_-generalized for unsaturated media combined with this functional relationship and substituted into the water conservation equation - determines the basic equation of liquid movement. This experiment determines the infiltration branch of the retention relation. An opposite test, starting with an initially liquid saturated column then allowed to drain down into a bottom dish, defines the socalled "drainage" branch of retention. Depending on which way the liquid is moving, either into or out of a volume of porous medium, then the infiltration or drainage branch equilibrium curves are employed with Darcy's law to calculate liquid movement. This is called accounting for hysteresis, because the infiltration and drainage retention relationships are generally different. Recall that during a spill situation, the entry of NAPL to the subsurface will follow the two stages: first infiltration then drainage. The drainage retention curve may be adequate to establish the final liquid content at the surface following a spill after sufficient time, if evaporation is a minor aspect for the particular liquid.

Lastly, note that upward imbibition, which was just described, and downward infiltration are both determined by the soil water retention. For infiltration, the capillary suction acts with gravity and not against it to draw liquid downward. So infiltration, which is the process connected with spreading, is driven downward by both gravity and the capillary suction into a soil.

\subsubsection{Intrinsic Permeability}

The cross-sectional area of the pore space permits flow through a porous medium. The liquid conductivity for each liquid involved can be derived by using this parameter in conjunction with the density and viscosity, and by using a relative conductivity function. This relative conductivity function is generally obtained theoretically using the water retention relation, which accounts for the distribution of pore sizes. The concept of intrinsic permeability (an area) presumes that any liquid moving in the same pore space will be affected by the same flow area. Multiphase flow theory provides a method for partitioning that area between the fluids that are present at each location, including the gas phase. Often, this parameter can be correlated with soil type or porous media kind and the bulk density. In addition, there are theoretical formulas for estimating intrinsic permeability from grain sizes. Generally, intrinsic permeability is measured directly for any porous medium. Bear's (1972) book deals with some of the various conceptualizations for constructing permeability from grain size information. A more extensive and updated explanation of this subject is given by Dullien (1992). Kao and Hunt (1996) provide a useful table of values related to particle sizes. The information is sufficient to cover a great variety of granular porous media.

\subsubsection{Grain Size Distribution}

Grain size, or particle size, distribution is the fractional distribution of each size of grain composing a granular porous medium. The method for measuring the grain size distribution is described by Gee and Bauder (1986). A particular difficulty with the spill problem is that the grain size distribution for surface material may not be the same as the subsurface. In fact, the surface is often composed of a skin-like crust that is quite distinct in hydraulic properties from soils a few centimeters deeper. This suggests it may be necessary to measure or characterize the surface material by performing specific measurements. 


\subsubsection{Vapor Diffusion Coefficient}

The vapor diffusion coefficient quantifies how much vapor flux can pass through the soil surface at a given vapor pressure gradient for a specific evaporative liquid. The point of evaporation of a liquid having a negligible vapor pressure may be well below the soil surface. In this case, the vapor diffusion coefficient describes the resistance to flow out of the soil surface. A fine-grained and packed surface material can greatly impede the evaporation, whereas a spill on coarse gravel gives little evaporation impedance. The amount of water present in the pore space can also modify the vapor diffusion parameter. For instance, if rainfall occurs after a NAPL spill, then the air-filled pore volume is reduced.

\subsection{Land Surface Properties}

There are physical properties and parameters associated with the macroscopic features of the land surface (see Table 3.3). These work in conjunction with the inherent properties of the liquids and porous medium to determine the outcome of a spill. Certainly such information would be needed to understand a spill pattern, even if a completely predictive model could not be constructed to quantify their influence. It is presumed here that overland flow theories for water can be appropriately modified to describe a NAPL. However, the particular NAPL may interact with the surface entirely different than water. In particular, a surface of clay material could transmit water and certain NAPLs entirely differently.

\subsubsection{Topography}

Topography is a geological map of elevation over the surface of concern. On a smaller scale relevant to a spill, even the number and sizes of boulders in the liquid path could be important in controlling surface dispersal. Small-scale topography features make it difficult to relate the surface pattern to the amount spilled.

\subsubsection{Roughness}

A spill will spread to a different extent if the surface is either smooth or rough with many surface depressions. Runoff models often incorporate such a parameter. Use of roughness and topography are described by Esteves et al. (2000).

Table 3.3. Spill Land Surface Parameters, Units, Symbols, and Sources of Information

\begin{tabular}{|l|l|c|l||}
\hline \multicolumn{1}{|c|}{ Parameter } & \multicolumn{1}{c|}{ Units } & Symbol & \multicolumn{1}{c|}{ Information Source } \\
\hline Topography & Elevation, $\mathrm{ft}, \mathrm{m}$ & - & USGS \\
\hline Roughness & - & - & - \\
\hline Macropore Distribution & Number per unit area & - & - \\
\hline Wind Speed & $\mathrm{m} \cdot \mathrm{s}^{-1}, \mathrm{~cm} \cdot \mathrm{s}^{-1}, \mathrm{ft} \cdot \mathrm{s}^{-1}$ & $\mathrm{U}, \mathrm{u}$ & Weather Stations \\
\hline Weather & Temperature, $\mathrm{RH}$, precipitation & $\mathrm{T}, \mathrm{RH}$, & Weather Stations \\
$\begin{array}{l}\mathrm{RH}=\text { relative humidity } \\
\text { USGS }=\text { U.S. Geological Survey }\end{array}$ \\
\hline
\end{tabular}




\subsubsection{Macropores}

Not all surface material is a simple unconsolidated granular medium. The surface may be cracked crust or rock with fissures or fracture channels. Large channels, wormholes, and plant root channels may be the major path for liquid flow into the ground in some locations. Other theory for liquid infiltration would have to be employed if this situation happens to prevail. Leonard et al. (2001) describe some of the concepts associated with water infiltration via macropores.

\subsubsection{Wind Over Land Surface}

Measurements of wind speed are usually required information for estimating evaporation of liquids. This is local information obtained from the weather forecast services.

\subsubsection{Weather}

This is all meteorological information such as temperature and rainfall amounts that could be needed to account for modification of a spill area. Wind speed is commonly available information, and a main factor controlling evaporation of a spill.

\subsection{Sources for Soil Properties}

There are three main functional relations for representing soil hydraulic properties in general use: 1) Brooks and Corey, 2) Campbell, and 3) Van Genuchten models. The parameter definitions for these unsaturated soil-water models are defined by Rawls et al. (1993). In addition, Rawls et al. (1993) provide correlations of the models with the soil types of clay, silt, and sand. Loamy soils, asphalt, and concrete will also need to be considered. These model parameters may potentially be modified to describe a liquid other than water by using the fluid-media scaling concept of the multiphase constitutive theory. Another more recent source of hydraulic properties is reported by Mohanty et al. (2002). A web site with a substantial listing of basic soil properties is available from Mohanty et al. (2002). This information can be combined with the liquid property data, as that provided by Mercer and Cohen (1990), to construct three-phase constitutive relations (retention and conductivity as functions of fluid saturations). In addition, Clapp and Hornberger (1978) give a simple list of common soil type and associated typical parameter values. The information is adequate for use with a simple spill screening model. 


\subsection{Available Spill Models}

The design and purpose of the identified models is discussed here. This search for spill models addressing specifically the first centimeter of land surface was not very successful for the reasons previously discussed. However, some partial models were discovered that could be useful to build a complete spill model. There are many codes that deal with ocean spills of petroleum, but ocean spills are not considered directly germane for land surface conditions. Further, ocean spills do not meet the needs for overland spill modeling. On the other hand, land surface spill models appear to be in the early stages of need recognition.

Adequate spill spreading models do not exist because developers have not yet attempted to couple overland flow models with multiphase subsurface flow simulators to address the behavior of a nonaqueous liquids running over and into the land.

In this section, a brief review of potentially useful models for spreading is given. It examines exactly what these spreading models are capable of and highlights some of the physical concepts involved. Some example modeling calculations for spreading of a spill on a porous surface are discussed. The example calculations required implementing simplified versions of the spill models and demonstrate aspects of coupling the spreading with infiltration.

\subsection{Screening Model for Surface Spreading}

One model was identified that deals with petroleum spreading on the land surface coupled with infiltration into the subsurface (Hussein et al. 2002). A code for this model was developed for the U.S. Environmental Protection Agency. However, the code apparently has not yet been released to the public. It may be obtainable from the consulting company involved in its development, but this is not certain. The model, however, is simple enough that it can likely be easily reproduced.

The Hussein et al. (2002) model uses gravity currents to describe the spreading and the Green-Ampt sharp front model to represent infiltration. A theory for spill evaporation is taken from the literature dealing mainly with spills on the sea. The evaporation component of the model does not treat the situation when vapors must diffuse from below the surface to escape. The roughness of the land surface is addressed as being storage depressions. Most restrictive, the model does not treat the decrease of NAPL content at the surface as a result of drainage. The lack of drainage is a serious limitation for estimating how long a spill might be detected just beneath the land surface.

\subsection{Models Treating Partial Problem Aspects}

\subsubsection{Infiltration of Nonaqueous Liquids}

There are many fully developed simulators for computing NAPL infiltration. Miller et al. (1998) reviewed many that are published and possibly available as computer codes. Pacific Northwest National Laboratory (PNNL) has available a code by Kaluarachchi and Parker (1989) that has a long history of use; it is called MOFAT-2D and has been tested and proven. This makes the code a strong choice as the 
basic component for describing the infiltration process. Although other published codes are just as capable, the important attributes are its availability, familiarity and public documentation.

The STOMP code is the most developed and diverse simulation tool available at PNNL (White and Oostrom 2000). It can be used to solve nearly any conceivable problem of subsurface movement of NAPL, including water-soluble and vapor phase transport as influenced by thermodynamic conditions. Most importantly, the code has an extensive history of verifications at PNNL. Thus the STOMP code would be appropriate for treating the general infiltration aspects associated with nearly any conceivable spill scenario.

\subsubsection{Over Surface Spreading}

A gravity current model by Acton et al. (2001) describes spreading with infiltration into an unlimited deep porous profile. The subsurface medium is presumed homogeneous, and the infiltration is supposed to occur by a saturated front. The solution is theoretically exact and could be used as a simplified evaluation tool. A computer code to solve the equations is discussed by the authors, but it is not known if it is available. The published description, however, is precise enough, so it can be reproduced. Its implementtation is discussed later in this report.

\subsubsection{Pool Formation and Spreading}

A new model was reported and derived from chemical engineering dealing with the spill of extremely hazardous liquids that react violently with water. The chemicals envisioned, however, would be considered miscible rather than immiscible liquids. The chemicals are also highly volatile when in contact with water. In addition, the forms of chemicals considered would likely be entirely transformed or reacted from their original chemical composition. Kapias et al. (2001) describe the model called REACTPOOL in a series of publications. The model conceptualizes a spreading pool of spilled liquid, and the authors suggest that it might be applied to any liquid spill. The spill spreading description, however, is highly simplified and disregards the influence of liquid viscosity, in particular. It does not account for penetration of the spill into a soil as spreading takes place. However, the model does consider a simple description of liquid permeating a concrete floor as well as spreading. Note that surface roughness is used to determine the final extent of a spill. Roughness determines a minimum depth for which spreading can continue. This approach is found to be a common aspect of all the spill models that were identified.

\subsubsection{Evaporation of Spills}

A model for simulating vapor emissions from multi-component liquid spills was created by Cavanaugh et al. (1993) of the Exxon Research and Engineering Company. LPOOL, the public domain version of the model, quantifies heat transfer to determine pool temperature and boiling status of the pool. The evaporative flux of a nonboiling pool is simulated by a method similar to that presented by Hussein et al. (2002), again lacking consideration of subsurface evaporative processes.

An organics behavior assessment model developed by Jury et al. (1983) simulates movement of organics incorporated into the soil. The analytical solution accounts for sorption, degradation, and 
enhanced volatilization due to soil water evaporation. The model requires quantification of the stagnant boundary layer thickness, which often requires micrometeorological data to determine accurately. The model assumes isothermal conditions.

Reinke and Brosseau (1997) developed a simplified spreadsheet model for predicting spill evaporation rates. The model allows for either isothermal conditions or prediction of spill temperature by way of a heat balance. The model assumes that as evaporation occurs, the pool maintains a constant depth and only decreases in area, a presumption that may be inaccurate for most permeable surfaces.

Yates et al. (2002) describe a one-dimensional numerical model developed to simulate pesticide degradation and volatilization into the atmosphere. Volatilization conditions may be varied to consider isothermal conditions, enhanced volatilization due to solar driven temperature changes at the soil surface, and volatilization from soil in relation to atmospheric processes such as wind speed. A code is not readily available and would likely have to be reproduced from the reference.

The multi-component water reactive spill model REACTPOOL (Kapias et al. 2001) contains a predictive evaporation component. The model accounts for heat balance to determine the boiling state of the pool. The significant difference between the evaporative component of REACTPOOL and LPOOL is that REACTPOOL incorporates turbulent diffusion into its simulation. The model treats the surface as impermeable.

\subsection{Spill Model Coupling Needs}

More advanced models for predicting patterns of overland flow were developed for surface water use (Esteves et al. 2000). Such models could be modified to account for different viscosity and interfacial tension if wanted. A surface flow model using the Saint Venant equations needs to be coupled with a more general NAPL infiltration code.

The spatial and temporal scales of the processes must be coupled appropriately to achieve accuracy. This is a specific mathematical development that needs substantial work emphasis. There are standard procedures to interface different codes so that the input and output is exchanged appropriately. A more mechanistically complex spill model, however, would require a considerably greater detail of input data. Section 3 lists input parameters and information that would likely be required to operate a more advanced model when it is developed.

\subsection{Simplified Spill Spreading Model}

A gravity current is a flow of liquid under a lighter fluid while moving along a surface. The flow is driven by the liquid's effective weight within the lighter fluid above it. For spills of liquids, the lighter fluid is usually only air. During spreading of a liquid, the forward movement is driven by gravity as the liquid collapses under its own weight, while at the same time viscous force resists the increase in speed of spreading. Huppert (1982) derived the following basic equation for a single dimensional current. 


$$
\frac{\partial}{\partial \mathrm{t}} \mathrm{h}-\frac{\rho \cdot \mathrm{g}}{3 \cdot \mu} \cdot \frac{\partial}{\partial \mathrm{x}}\left(\mathrm{h}^{3} \cdot \frac{\partial}{\partial \mathrm{x}} \mathrm{h}\right)=0
$$

where $h(x, t)=$ height or head of the liquid, depending on density

$$
\begin{aligned}
& \rho= \\
& \mu=\text { viscosity } \\
& g=\text { acceleration of gravity }
\end{aligned}
$$

Note that Equation (4.1) is a form of nonlinear diffusion equation for which the effective diffusion coefficient depends on the third power of $h$. This effective diffusion is proportional to the factor

$$
\mathrm{R}=\frac{\rho \cdot \mathrm{g}}{3 \cdot \mu}
$$

The $\mathrm{R}$ in (4.2) (see Table 4.1) carries the physical attributes of the liquid that determine how fast it will spread as the head $h(x, t)$ redistributes under the influence of the effective diffusion. The importance of (4.1) is that the equation expresses how boundary conditions on the spreading flow can be incorporated into a particular problem.

Huppert (1982) solved (4.1) under the boundary constraint that

$$
\int_{0}^{x_{N}(t)} h(x, t) d x=q \cdot t^{\alpha}
$$

where

$$
\mathrm{q}=\text { input rate at the origin, } \mathrm{x}=0
$$

$\mathrm{x}_{\mathrm{N}}(\mathrm{t})=$ locates the front edge as depends on time, $\mathrm{t}$.

Note that $\alpha$ is a parameter that allows for a variety of input situations. Most importantly, $\alpha=0$ is an instantaneous input, whereas $\alpha=1$ is linear dependence on time. These different input conditions for various $\alpha$ values are important for different spill release occurrences.

Huppert's (1982) solution of (4.1) subject to (4.3) is analytical and gives the front location as

$$
x_{N}(t)=\eta_{N} \cdot\left(R \cdot q^{3}\right)^{\frac{1}{5}} \cdot \frac{(3 \cdot \alpha+1)}{5}
$$

Note how the front distance (4.4) depends on $\mathrm{R}$, which has units of $1 /(\mathrm{cm} \mathrm{s})$, so the effective diffusion coefficient that drives $h(x, t)$ has units $\mathrm{cm}^{2} / \mathrm{s}$. The value of $\mathrm{R}$ for water is $\mathrm{R}=32,700 / \mathrm{cm} \mathrm{s}$. Values of $\mathrm{R}$ for 
other liquids that could be involved in land spills can be readily compiled for use in (4.4). Equation (4.4) is a first simple model for spill spreading. It would apply when the surface is not permeable to any extent. The parameter $\eta_{N}$ depends on $\alpha$; Huppert provides a graph for its values. It equals 1.411 for $\alpha=0$ and 1.01 for $\alpha=1$. Its value changes little after $\alpha$ of $1 / 2$, for which it is 1.10 . For most spill problems, its slight difference is unimportant to gauging the extent of spreading.

Equations (4.1) and (4.4) would apply to a spill running off a straight edge of a highway or pavement. However, Huppert (1982) also derived similar equations for a radial geometry involving two-dimensional spreading from a central point source. The radial spreading equation is

$$
r_{N}(t)=\xi_{N} \cdot\left(R \cdot Q^{3}\right)^{\frac{1}{8}} \cdot \frac{(3 \cdot \alpha+1)}{8}
$$

where $\mathrm{r}$ is the radius. Now $\mathrm{Q}$ is a volume input instead of the liquid area, $\mathrm{q}$, as is defined for the case in (4.4). The head, $h$, for radial coordinates is subject to a constraint like (4.3), but integrated instead over expanding rings of circular area.

Programs were devised using Mathcad software to calculate gravity currents according to Huppert's analytical derivations. The importance of such programs is that they are used to verify calculations when the spreading is coupled with infiltration into a porous surface.

Hussein et al. (2002) recently recognized the relevance of Huppert's work for estimating the surface spreading of a spilled petroleum liquid on the land. These authors also identified the relevance of Lister's (1992) derivations for gravity currents flowing down an inclined surface. Again, a Mathcad program was devised to implement inclined gravity currents so to determine how such flow would develop and differ from a level surface. Although Hussein et al. (2002) review the partial differential equations for a gravity current on an incline, for line and point sources, they do not attempt to solve them for the head distribution in space as depends on the time. On an inclined surface, the flow would move mainly downhill, but would also move slightly uphill as well as transverse outwardly.

Because Hussein et al. (2002) were apparently only interested in deriving a simplified screening model for a liquid spill, they only made use of equations such as (4.4) and (4.5) for estimating the extent of the spreading. A screening model for spill spreading requires coupling the liquid infiltration into the porous land surface as the liquid spreads. The phenomenon of infiltration, which is described mathematiccally by Darcy's law for liquid conduction in a soil medium, was described previously. The infiltration process is driven by the overlying head of liquid during a spill and is complicated because the absorption of liquid into the subsurface soil happens as an unsaturated flow process. In any case, to model or estimate the extent of spill spreading, it is necessary to predict or estimate the influence on the spreading speed of liquid removal by infiltration. Clearly, as infiltration occurs while removing liquid head, the spreading is compelled to slow down.

Hussein et al. (2002) modified Equations (4.4) and (4.5) to depend explicitly on the liquid volume present at any moment. This modified front equation was coupled with infiltration removal of a liquid to 
estimate how spreading would progress. There is no exact mathematical justification for their alteration of the front equations, except that it is a conceptually reasonable approach to avoid solving the complicated partial differential equation for a gravity current, when coupled exactly to an infiltration sink at each location in space. However, they did demonstrate that the proposed volume-dependent extension of the spreading front equation would reproduce the spill spreading correctly when there was no loss of liquid volume involved.

A similar simplified screening model was devised for this work, because the code by Hussein et al. (2002) could not be obtained. A different equation for spreading front movement, however, was derived. Instead of depending only on the liquid volume currently present, a model was derived to depend on both the prevailing liquid head or height, $\mathrm{h}$, and the present front extent. Here is how the simplified spreading is conceptualized.

Let $\mathrm{H}$ denote an average value of $\mathrm{h}$ over the spreading region. In general, the shape of the gravity current, h, would change; it is the forward tilt or decline in head that causes the current to advance according to Equation (4.1) as a diffusion process. The conservation of liquid above the surface is expressed as follows:

$$
x_{N}(t) \cdot H(t)=q \cdot t^{\alpha}
$$

The value of $q$ in (4.6) is presumed to apply only momentarily to the amount of liquid that is currently above the surface. Thus q must decrease over time as liquid is removed from the actual amount spilled. It is still supposed that (4.4) or (4.5) will apply for this momentary q, describing only the liquid on the surface. Note that there would be one fixed value of "q" that would define how rapidly the entire spill occurred as it flowed over and into the land surface. Substituting (4.6) for this effective q into (4.4) gives

$$
\mathrm{x}_{\mathrm{N}}(\mathrm{t})^{2}=\eta_{\mathrm{N}}^{5} \cdot \mathrm{R} \cdot \mathrm{H}^{3} \cdot \mathrm{t}
$$

Notice (4.7) suggests that the square of the spreading distance is directly proportional to the effective spreading diffusion coefficient and the time. On the other hand, if the change in the effective q with time is neglected, then Equation (4.4) or alternatively 4.5 can be differentiated with respect to $t$ to obtain a front velocity given by

$$
V_{N}(t)=\frac{d}{d t} x_{N}(t)=\frac{(3 \cdot \alpha+1)}{5} \cdot \frac{x_{N}(t)}{t}
$$

Now substituting (4.8) into (4.7) for $t$ gives

$$
\mathrm{V}_{\mathrm{N}}(\mathrm{t})=\left(\frac{3 \cdot \alpha+1}{5}\right) \cdot \frac{\eta_{\mathrm{N}}^{5} \cdot \mathrm{R} \cdot \mathrm{H}^{3}}{\mathrm{x}_{\mathrm{N}}}
$$


The extension of the theory is that (4.9) should apply depending only on prevailing values of $\mathrm{H}$ and $\mathrm{x}_{\mathrm{N}}$, regardless of how much liquid is being input by spill release or extracted by infiltration. However, (4.9) still reflects the rate of input through $\alpha$ and parameter $\eta_{\mathrm{N}}$. After the input is completed over a finite period, $\alpha$ is changed to zero to indicate that the amount remaining on the surface was released as if instantaneously and then moved across the surface to the current spreading front location. In any case, the velocity of advance by (4.9) depends on both of the average head, $\mathrm{H}$, and front distance, $\mathrm{x}_{\mathrm{N}}$, not on just the present surface volume of spilled liquid.

Some typical values for R in (4.9) are given in Table 4.1. In Table 4.1, the values for many fuels and oils are not greatly different than for water. Some are much more viscous and slower flowing too.

The simplified spill spreading model is implemented as follows on a distance range, L. L is divided into $\mathrm{N}$ intervals, each with size $\mathrm{dX}=\mathrm{L} / \mathrm{N}$. At each time step when the preceding $\mathrm{H}$ and the spill extent, $\mathrm{X}$, are known, the velocity (4.9) estimates how long, dT, it will take the spill to advance $\mathrm{dX}$. After the spreading is advanced to $\mathrm{X}+\mathrm{dX}$, the infiltration removes liquid during the time $\mathrm{dT}$. A new value of $\mathrm{H}$ is then found based on the amounts input by the spill release and removed by infiltration. That is, the liquid conservation equation becomes

$$
x_{N}(t+d T) \cdot H(t+d T)-x_{N}(t) \cdot H(t)=q \cdot(t+d T)^{\alpha}-q \cdot t^{\alpha}-I
$$

Table 4.1. Values for the Parameter $R$

\begin{tabular}{|l|c|c|c|c||}
\hline \multicolumn{1}{|c|}{ Liquid } & $\begin{array}{c}\text { Temperature } \\
\left({ }^{\circ} \mathbf{F}\right)\end{array}$ & $\begin{array}{c}\text { Specific } \\
\text { Gravity }\end{array}$ & $\begin{array}{c}\text { Viscosity } \\
(\mathbf{c p})\end{array}$ & $\begin{array}{c}\text { R parameter } \\
\mathbf{( 1 / c m ~ s )}\end{array}$ \\
\hline Water & 60 & 0.999 & 1.13 & $2.89 \times 10,000$ \\
\hline Solvent & 60 & 0.721 & 0.92 & $2.57 \times 10,000$ \\
\hline Carbon tetrachloride & 60 & 1.595 & 1.04 & $5.03 \times 10,000$ \\
\hline Med Lube Oil & 60 & 0.896 & 156.5 & 187.2 \\
\hline Med Lube Oil & 40 & 0.905 & 401. & 73.8 \\
\hline Med Fuel Oil & 60 & 0.858 & 3.8 & $7.41 \times 1,000$ \\
\hline Reg Gasoline & 60 & 0.728 & 0.49 & $4.82 \times 10,000$ \\
\hline Reg Gasoline & 100 & 0.71 & 0.40 & $5.86 \times 10,000$ \\
\hline Kerosene & 68 & 0.809 & 1.73 & $1.53 \times 10,000$ \\
\hline Turpentine & 68 & 0.862 & 1.49 & $1.89 \times 10,000$ \\
\hline Ethyl Alcohol & 68 & 0.789 & 1.21 & $2.13 \times 10,000$ \\
\hline Benzene & 68 & 0.879 & 0.655 & $4.39 \times 10,000$ \\
\hline Glycerin & 68 & 1.262 & 834 & 49.5 \\
\hline Machinery Oil & 62 & 0.907 & 124 & 239. \\
\hline Transmission Fluid & 68 & 0.84 & 71 & 387. \\
\hline $\begin{array}{l}\text { Med = medium } \\
\text { Reg = regular }\end{array}$ & & & & \\
\hline
\end{tabular}


where I is the infiltration amount removed over all the intervals $\mathrm{dX}$ where liquid will be present at the end of each time step. Here q in (4.10) is the fixed value that is controlling the spill release rate, not the changing effective value used in deriving Equation (4.9). Note that at the end of the release period (assumed finite), the alpha is changed to zero, and then only infiltration is removed from the remaining surface amount until it is gone into the subsurface.

A simplification of Darcy's law and a solution for infiltration called the Green-Ampt formula can be applied to obtain I in (4.10). Hussein et al. (2002) and Acton et al. (2001) both have used the GreenAmpt model to describe infiltration. The infiltrating flux, $\mathrm{f}$, of liquid is

$$
\mathrm{f}=\mathrm{K} \cdot \frac{\left(\mathrm{h}-\mathrm{H}_{\mathrm{f}}+\mathrm{Z}\right)}{\mathrm{Z}}
$$

where

$$
K=\frac{k \cdot \rho \cdot g}{\mu}
$$

where $Z=$ front depth from the surface

$\mathrm{h}=$ liquid head above surface

$\mathrm{H}_{\mathrm{f}}=$ pressure head at the front.

$\mathrm{H}_{\mathrm{f}}$ is a negative pressure meaning a capillary suction that draws liquid into the porous medium under unsaturated conditions. For an immiscible liquid other than water, the permeability, $\mathrm{k}$, being the product of intrinsic permeability and a relative value based on the available pore volume is dependent on the initial volumetric content of water. That is, presuming that water would be the more wetting liquid, the remainder of the porosity not occupied by water could be filled with the spilled liquid. Note that multiphase flow theory provides equations to calculate the permeability for the spilled immiscible liquid relative to water.

Let $\phi$ be the porosity available to the spilled liquid. Then, the Green-Ampt formula is obtained from the following:

$$
\begin{gathered}
\phi \cdot \frac{\mathrm{d}}{\mathrm{dt}} \mathrm{Z}=\mathrm{t} \\
\mathrm{t}-\mathrm{t}_{\mathrm{o}}=\frac{\phi}{\mathrm{K}} \cdot\left(\mathrm{Z}-\mathrm{Z}_{\mathrm{O}}-\mathrm{h}^{\prime} \cdot \ln \left(\frac{\mathrm{h}^{\prime}+\mathrm{Z}}{\mathrm{h}^{\prime}+\mathrm{Z}_{\mathrm{O}}}\right)\right)
\end{gathered}
$$

where $\quad h^{\prime}=\mathrm{h}-\mathrm{H}_{\mathrm{f}}=$ total pressure head causing infiltration 


$$
\mathrm{Z}_{\mathrm{O}}=\text { depth where liquid is already infiltrated prior to the initial time, } \mathrm{t}_{\mathrm{o}} \text {. }
$$

Equation (4.13) is derived assuming that $\mathrm{h}$ remains nearly constant over the period of integration of (4.12). The amount of liquid infiltrated during a time step $d T=t-t_{0}$ is $\phi \cdot\left(Z-Z_{0}\right) \cdot d X$ within each interval of size $\mathrm{dX}$. Here $\mathrm{Z}$ depends on location $\mathrm{X}$ where the head $\mathrm{h}$ is taken as the average $\mathrm{H}$ over the entire spreading distance $\mathrm{x}_{\mathrm{N}}$. Thus, the simplified screening model uses the imposed condition that $\mathrm{h}$ is uniformly equal to $\mathrm{H}$ at each time, and the exact distribution of $\mathrm{h}$ that would be given by solving (4.1) is not considered.

Note that $I$ in $(4.10)$ is

$$
I=\sum_{d X} \phi \cdot\left(Z-Z_{0}\right) \cdot d X
$$

which is the sum of all infiltrations over the spreading region after each time step.

In general, the calculation of infiltration is a complicated nonlinear problem as discussed by Haverkamp et al. (1977). The calculation would be computationally intensive if combined directly with (4.1) as a sink term put on the right-hand side of the equation, replacing the zero. Thus, the simplification of both the spreading as a gravity current and unsaturated liquid infiltration as a Green-Ampt wetting front is done to reduce computational burden and make the spill spreading problem easier to solve. However, without exact experimental confirmation, it is not at all clear what the accuracy of this simplified model for a spill would be. Only the two subprocess models for gravity spreading and infiltration can be tested separately to assess the possible degree of accuracy.

For instance, the Green-Ampt model can be compared with a precise simulation of infiltration into a definite soil. Figure 4.1 shows how the model compares with ponded infiltration into a well-known soil named Yolo light clay. The hydraulic properties of Yolo Clay compared with a fine sand are given in Haverkamp et al. (1977).

To determine the Green-Ampt wetting front depth, the pressure head at the front was calculated using the method derived by Neuman (1976). A suction pressure of $26.5 \mathrm{~cm}$ is added to the $25 \mathrm{~cm}$ of applied pressure head to calculate the wetting front depths. Weaver et al. (1994) as cited by Hussein et al. (2002) discuss how the front pressure head is to be estimated for any immiscible liquid entering an already water wetted medium. Figure 4.1 shows that the sharp front Green-Ampt model is reasonably accurate even for a clay soil for which infiltration fronts are not actually sharp. The model is known to be more accurate for sand soils.

\subsection{Example Spill by Simplified Model}

The simplified model combines the simplified gravity current description with Green-Ampt infiltration model; Equations (4.9) and (4.13) are coupled. The validity of (4.9) can only be proven mathematiccally for the case when there is negligible infiltration. This is done to demonstrate the stepwise 


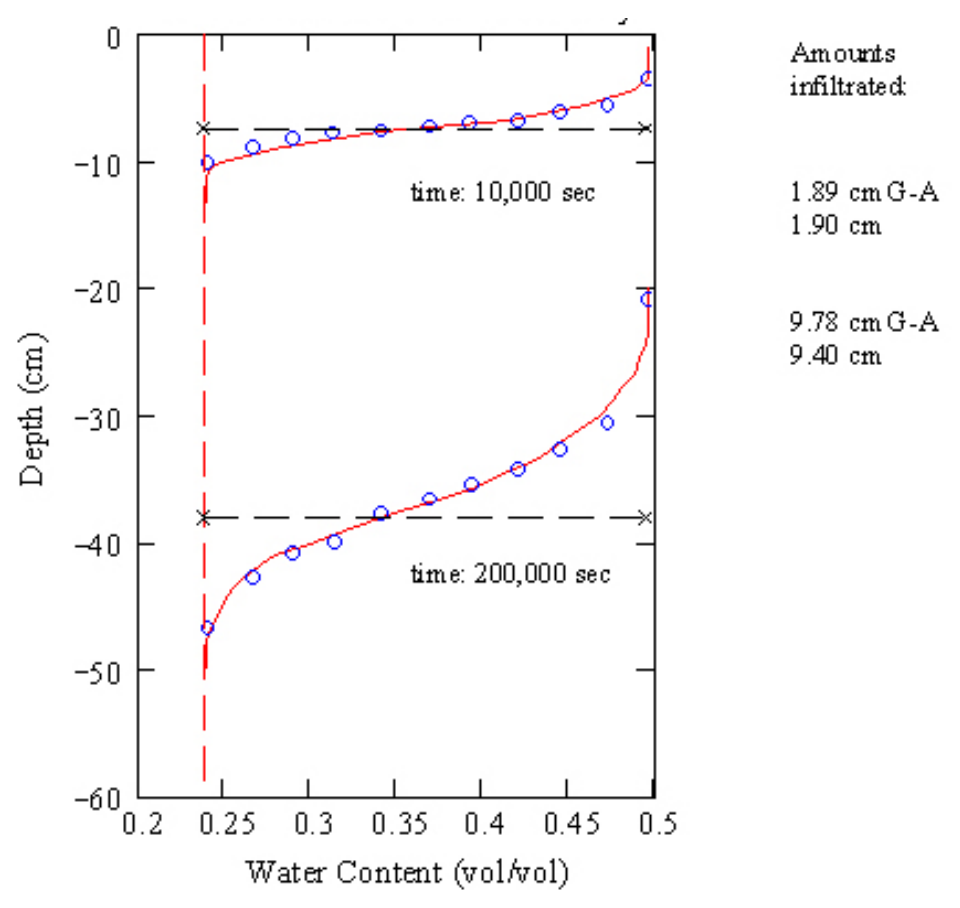

Figure 4.1. Infiltration into Yolo Light Clay Soil under Ponded Condition. (Applied pressure head $=+25 \mathrm{~cm}$ ). Horizontal dash lines for $10,000 \mathrm{~s}$ and $200,000 \mathrm{~s}$ indicate location of saturated Green-Ampt (G-A) fronts. Solid curve infiltration profiles were produced by unsaturated flow simulations (Haverkamp et al. 1977), and circles are analytical results obtained by Philip (1969).

integration of the front movement. The physical properties of water are used for reference. Consider a spill of 10 gal $(37,850$ cubic $\mathrm{cm})$ released over each $1 \mathrm{~m}$ length of line source, in $10 \mathrm{~s}$ at constant rate, for $\alpha=1$.

Figure 4.2 demonstrates the accuracy of the front tracking integration. The spill is released over $10 \mathrm{~s}$ with height following the $\alpha=1$ curve and then following the $\alpha=0$ curve as spreading continues without any further water input.

If input continued at the same rate beyond $10 \mathrm{~s}$, then the front would remain following the red curve (alpha 1) shown in Figure 4.2. The spill height is indefinitely large initially if released instantaneously, indicated by the dash curve (alpha zero).

Figure 4.3 presents the corresponding location of the spreading front depending on time.

Having proven the front tracking in $\alpha=0$, the absence of infiltration, the model can be applied to a case when the surface is permeable. Note that it would be appropriate also to test the infiltration calculation by finding the smallest spreading extent or spill surface area over which the spill can be evenly distributed without a head building up as water is applied. This would be the case where the spreading is presumed to occur instantaneous reaching a known distance. If the extent of applied liquid 


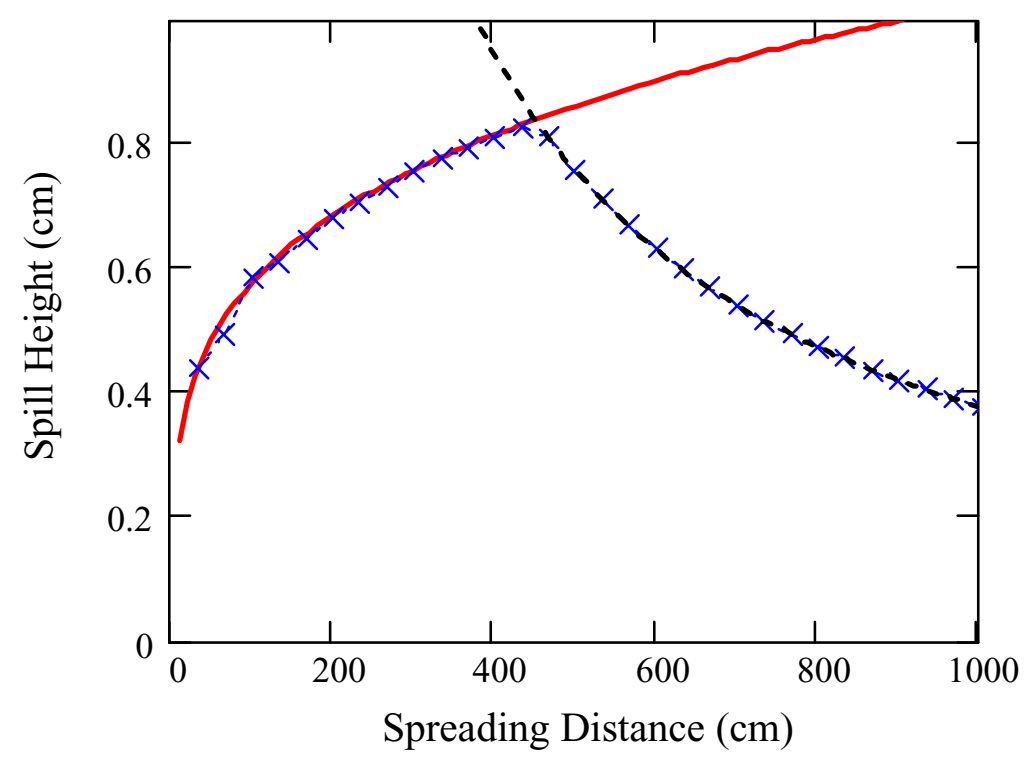

Figure 4.2. Average Spill Head for a Line Source by Simple Model. Water released is $10 \mathrm{gal} / \mathrm{m}$ of source length over $10 \mathrm{~s}$ without any infiltration. Solid red curve is the continuous release at the same rate and black dash curve is spreading of the spill for an instantaneous release. $X$ marks spreading progress as determined by integration of Equation (4.9) describing the front velocity.

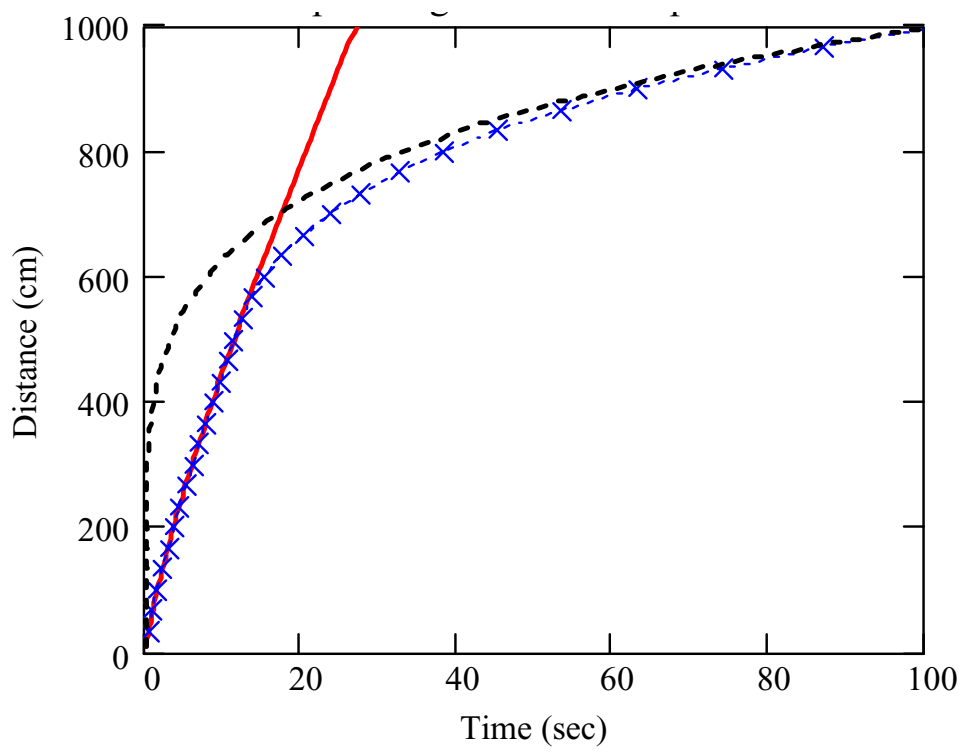

Figure 4.3. Spill Spreading Front Distance by Simple Model. Spill is $10 \mathrm{gal} / \mathrm{m}$ of source length over $10 \mathrm{~s}$. Curves correspond to those of Figure 4.2. 
were sufficiently small, as when confined by a mound barrier, then the water height would rise before finally gradually infiltrating entirely. Such a test was done also to prove the time step integration of the Green-Ampt model for infiltration. The time step integration was proven to reproduce the continuous solution of the Green-Ampt model for a time dependent input, for which the standing head is varying.

Figure 4.4 shows the conceptualized cross section of a spill on land with a permeability approximately equal to sand or gravel. It is the liquid distribution just after the release is finished. Note that the spill height is level over the spill extent as imposed conceptually in the simplified model.

However, the profile of liquid distribution below the surface indicates that the infiltration has progressed longer with greater head near the release location at zero distance.

Figure 4.5 provides the corresponding complete history of the spill height as it spreads and infiltrates. It shows that the spill disappears abruptly into the highly permeable subsurface $\left(\mathrm{k}=1 \mu \mathrm{cm}^{2}\right.$ and porosity $=0.4$ ), when the release is complete after $10 \mathrm{~s}$. Notice that in Figure 4.2, the spill without infiltration reached a height of about $0.8 \mathrm{~cm}$ before declining. This spill disappears in about $14 \mathrm{~s}$ into the subsurface. Its rather minor height is mainly a function of the slow release and not the infiltration. A much faster release would be required to create a height of a few centimeters.

To compare permeability, typical sand has a value of one-half that used for the simulation. The Yolo light clay soil described in Figure 4.1 is only 0.000125 of the $1 \mu \mathrm{cm}^{2}$. Thus, the infiltration into the Yolo Clay took considerably longer and would have been negligible for this simulated spill if it occurred on Yolo Clay.

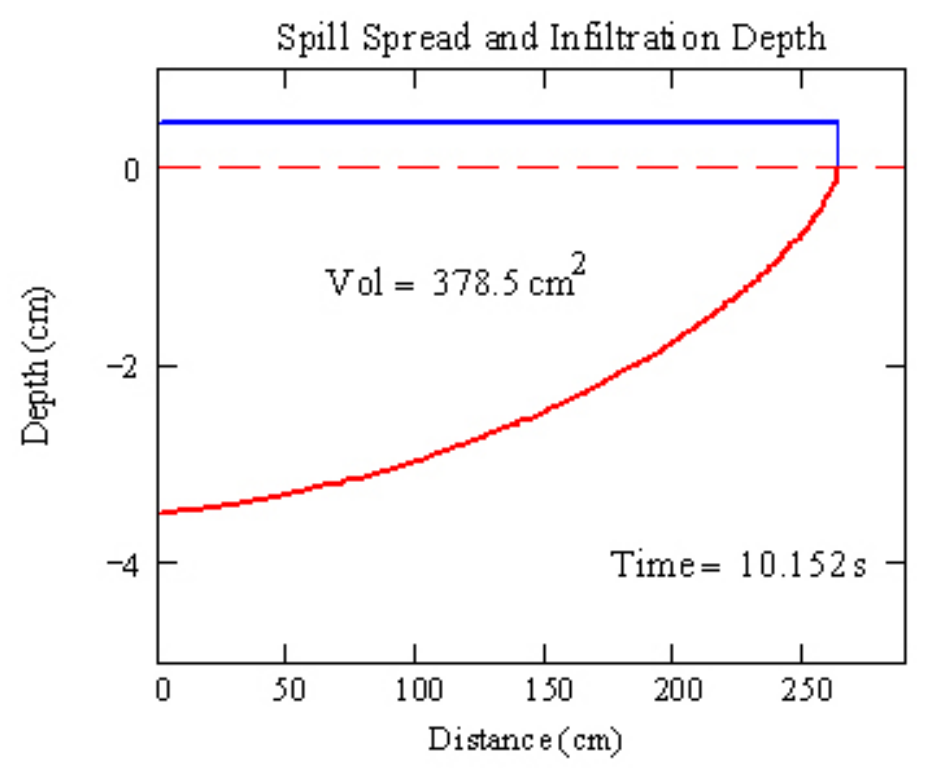

Figure 4.4. Spill Profile by Simplified Model. The volume is the area per centimeter of line source. Spill is $10 \mathrm{gal} / \mathrm{m}$ per $10 \mathrm{~s}$. Permeability is sand or gravel $\left(1 \mu \mathrm{cm}^{2}\right)$. 


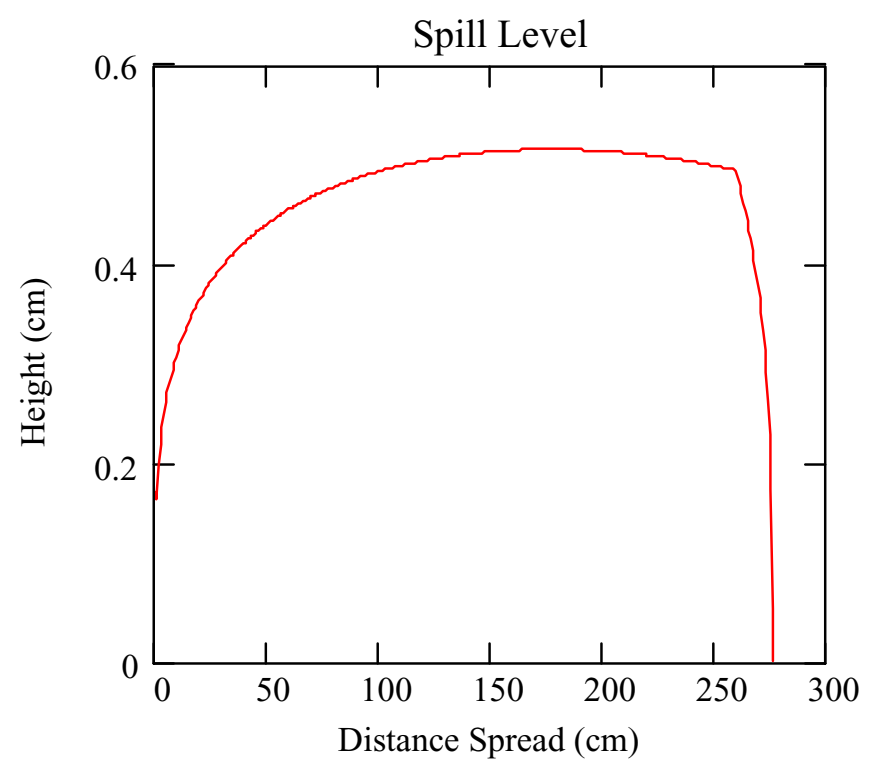

Figure 4.5. Spill Height by Simplified Model. Spill amount is $10 \mathrm{gal} / \mathrm{m}$ per $10 \mathrm{~s}$.

\subsection{An Exact Spill Model}

Acton et al. (2001) continued the research of Huppert on gravity currents and coupled the Equation (4.1) with saturated infiltration into a porous medium. Apparently, Acton et al. (2001) were not aware that the infiltration equation they used was actually the Green-Ampt model, without including a term for the wetting front pressure head. It is a simple modification of the Acton et al. (2001) derivation to include the full Green-Ampt model. The work done by Acton et al. (2002) was found independently of the model developed by Hussein et al. (2002). At least, Hussein et al. never cited those authors more recent work, even though it was exactly relevant. Moreover, the work by Acton et al. (2001) was a careful scientific study supported by laboratory experiments. Thus, their physical theory was confirmed by specific experiments with a viscous liquid flowing over a relatively high permeability medium. They began their discussion with a further demonstration that the Green-Ampt model is quite accurate and appropriate for the porous medium used in their fluid dynamical study.

Rather than developing an approximation, Acton et al. (2001) solved directly the partial differential equation of spreading coupled with infiltration. Their solution, however, was limited to special cases of release ( $\alpha=3$, allowing a similarity solution, and $\alpha=0$, for an instantaneous release). Most significantly, Acton et al. (2001) demonstrated that there is essentially only one pair of equations that requires solving. However, the equations do still depend on the one parameter of porosity. The gravity spreading and infiltration can be expressed by dimensionless variables for distance, time, liquid height, and infiltration depth. Thus, every solution associated with various boundary conditions, fluid properties, and soil permeability can be deduced from one solution obtained in terms of the dimensionless variables and the particular porosity.

Following their equations, a more direct mathematical method was applied to construct an exact model for spill spreading. Specifically, an explicit finite difference method was used to solve the coupled 
gravity current and infiltration equations as given by Acton et al. (2001). Such implementation allows for more general boundary conditions on the release pattern. For instance, an initial spreading distribution can be accommodated as when making a transition from a given liquid input rate to none entering.

The difficulty of using this more exact model rather than the simplified one is it is much more computer intensive and sometimes difficult to control convergence. Although the simplified model is not an exact solution of the theory, it is nevertheless much more flexible in allowing for easy modification to include other phenomena as is necessary. For example, it might be required to include loss of surface liquid by evaporation as well as by infiltration, if evaporation is expected to have a substantial impact on spreading behavior. Certainly, the Acton et al. (2001) work makes the important contribution of testing the accuracy of any simplified spreading model.

Figure 4.6 shows the calculated profile for the same release used to demonstrate the simplified spill spreading model. The maximum height of the surface liquid is $0.89 \mathrm{~cm}$ with a depth of $3.8 \mathrm{~cm}$ at the release point. Note that at the end of the release period, $10 \mathrm{~s}$, the distribution is tilted toward the front. It is the slope of the liquid surface that drives the gravity current forward. This detailed feature is not calculated in the simplified model. After the release period, the surface liquid height begins to flatten out and the front recedes as all liquid enters the subsurface.

Figure 4.7 shows that after the spill, the spreading distance actually reduces as liquid is infiltrated. It indicates the spill disappears from the surface after about $16 \mathrm{~s}$. For $2 \mathrm{~s}$ following the release, the spreading apparently nearly stands still, until receding. The spill did not spread beyond about $250 \mathrm{~cm}$. The exact spreading calculation indicates that the simplified model estimation was reasonably accurate.

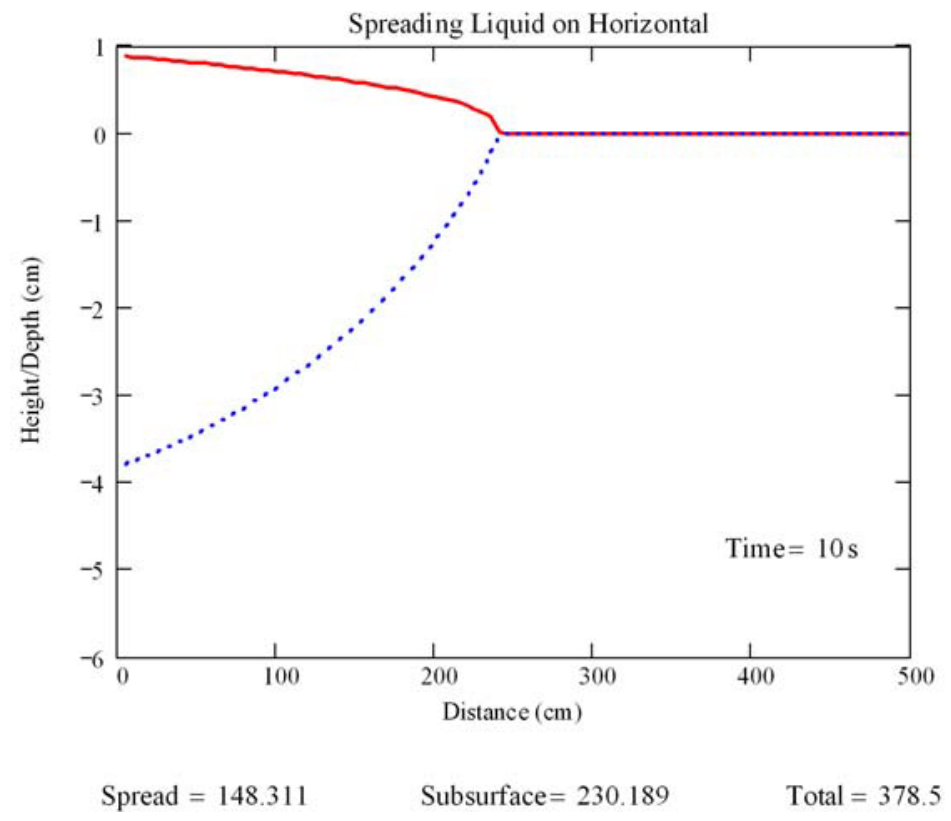

Figure 4.6. Spreading and Infiltration of a Spill on a Flat Surface. The numbers indicated as spread and subsurface are the amounts located above the surface and below following the spill. Solution is based on theory of Acton et al. (2001). 


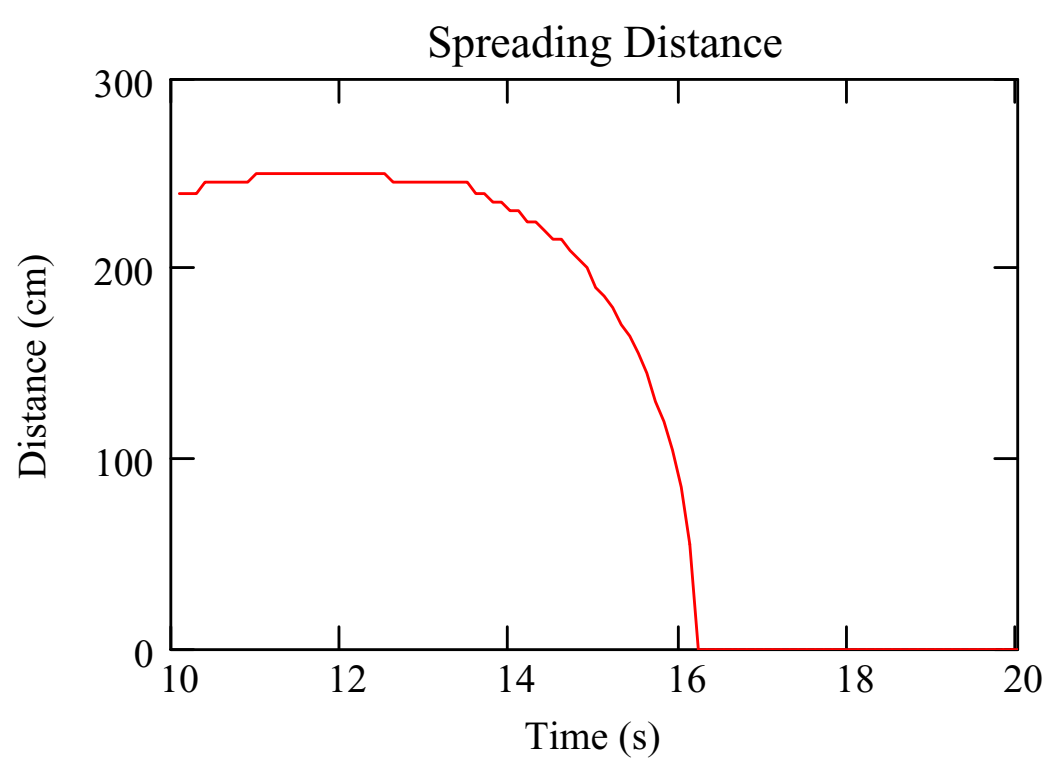

Figure 4.7. Spreading Distance After Spill on a Flat Surface

\subsection{Surface Roughness}

The derivation of Acton et al. (2001) presumes a very smooth surface without having any grooves or depressions (furrows) that would contain liquid as it spreads over a porous base. It is not unusual to imagine that a true field situation could involve a surface with $1 \mathrm{~cm}$ or even deeper groves in the ground. This could occur even if the surface is still considered level overall and conforms to the assumption of the horizontal spreading model. Under such a situation, the example gravity current that was modeled as resulting from a 10 -s duration release of $10 \mathrm{gal} / \mathrm{m}$ would not actually be able to propagate, because its height is always less than $1 \mathrm{~cm}$, for instance see Figure 4.5. Thus, the previous gravity current example would seem rather irrelevant to a realistic field situation (arbitrarily long groves closely spaced so that the liquid volume would be contained). Of course, the logical difficulty here is the supposed scale of the spilled quantity, when viewed relative to the depth of the surface grooving that could impede surface spreading.

To make the gravity current model of spreading more realistic, a certain amount of spill liquid would have to go into storage in the surface grooves. Only the liquid above the groove tops would be subject to gravity current flow. Hussein et al. (2002) use this conceptualization in their screening model. A benefit of the simplified spreading model is that it is easily adjusted to accommodate having a certain amount of spill go into retention or storage by surface grooves. Including this aspect in a partial differential equation description of gravity current is not as simple.

On the other hand, the spill release boundary condition can alter the importance of roughness. Consider instead an instantaneous release of $10 \mathrm{gal} / \mathrm{m}$. This problem, which corresponds to the case of $\alpha=0$, is more computationally demanding to solve, because greater head gradients are involved. Roughness becomes less of an influence because the height of the current remains greater. Figure 4.8 shows the result of this change in spill release rate. 


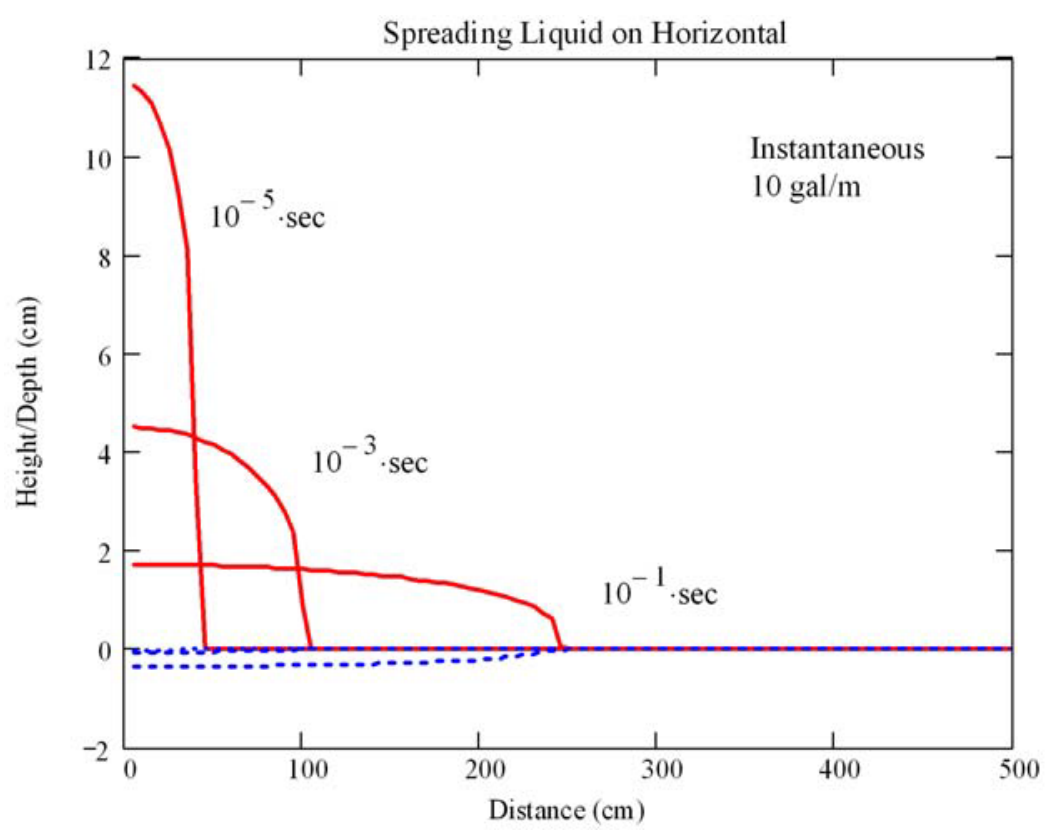

Figure 4.8. Spill Spreading for an Instantaneous Release. Blue dash line is liquid depth.

In a mere one-tenth of a second, the spill has reach $2.5 \mathrm{~m}$ and is higher than a nominal groove depth of $1 \mathrm{~cm}$. In another second, the spreading, however, would not be able to pass over this groove depth. Also, notice that the spreading is so rapid that not much liquid enters the ground. In contrast, in Figure 4.6, it took $10 \mathrm{~s}$ for the distributed release to reach about the same distance, but the liquid height could not have passed over 1-cm-deep grooves, if they were sufficiently numerous to contain the volume.

An adequate spill spreading model, therefore, needs to include roughness. It could be modeled as a grove-to-grove transfer of a liquid spill or as a continuously distributed sink removing liquid from the flow. The best method to account for roughness needs to be adapted to the kind of spreading model.

\subsection{Model Limitations}

The two spill spreading models (screening and exact) examined here are helpful to quantify some of the main phenomena, and they demonstrate the important coupling between overland spreading and infiltration. However, they both neglect other important mechanisms that could substantially impact spreading behavior. A list of limitations is given below. These suggest why other more complex models would need to be implemented to fully predict spill spreading. In particular, these models:

- Lack influence of surface slope and detailed topography

- Neglect infiltration of immiscible liquid as subject to water movement

- Neglect lateral horizontal infiltration by being only vertical

- Lack description of subsurface layers restricting infiltration 
- Lack a full description of fluid dynamics of surface flow based on gravity currents

- Fail to address long-term drainage/evaporation of the surface liquid content.

Surface slope is addressed somewhat by the screening model of Hussein et al. (2002) but only approximately. A sloping topography is likely to be more the rule than the special situation of a flat porous surface. Thus, the simple models discussed are too simple for general situations.

Infiltration of an immiscible liquid is greatly dependent of the presence of water and its concurrent movement when a liquid with different physical properties enters the ground. The models described here can be modified slightly to account for the presence of a uniformly static water phase that removes pore space available to an immiscible liquid's flow. Conceptually, this is important because water will keep another liquid from being absorbed into a wet or saturated soil. A more general multiphase flow simulator is required to replace the Green-Ampt model of infiltration.

Infiltration is not always mainly vertically downward; it depends on the degree of lateral absorption of liquid as well. The presence of sand layers just below the surface can modify the coupling between surface spreading and infiltration. An impermeable layer (clay) just below a sandy surface can cause it to remain liquid saturated following a spill, instead of draining. For usual land surfaces, infiltration is a three-dimensional process-not simply downward.

A limitation of any model-regardless of how mathematically complete it may be-is information about the structure and properties of the subsurface, which control the infiltration. A hypothetical description of overland flow might be inaccurate for real situations. Consideration of roughness is a challenging problem.

Gravity currents do not constitute by any measure a full representation of the fluid dynamics of overland flow. Unstable surface flow phenomena occur that require much more detailed physical theory. It is supposed that the Saint Venant equations would provide a more general basis when combined with a description of topography. However, strong or rapid spills may be dominated by turbulent and unstable flow processes, which depend on the shear behavior of the liquid involved. In other words, gravity current theory may work for very viscous liquids, but is it accurate for liquids such as gasoline, with a density and viscosity much less than for water?

The spill models examined here did not address drainage and evaporation. The Hussein et al. (2002) model, however, does give considerable attention to long-term evaporation processes. A more general subsurface flow model, going beyond the limitation of the Green-Ampt infiltration, would treat drainage over time as well. Clearly, whether a spill can be detected may depend more on how quickly it drains entirely from the surface, rather than on its spreading extent. 


\subsection{Conclusions on "State-of-the-Art" of Spill Modeling}

This study found that there are no completely developed computer models for treating problems of NAPL spill spreading over the land surface. Highly developed models and computer codes exist for treating overland water flow and multiphase subsurface flow separately, but standard techniques and procedures for coupling these two main aspects that determine the extent of spreading have not been implemented as yet. Simplified screening models or mathematical solutions applicable for developing such models were identified. However, codes were not made available openly to test or apply such screening models to real spill situations. Consequently, it has been necessary to implement some of the theory in simple computer models and programs to test its relevancy to answering basic phenomenologycal questions about spill behavior. Future spill models will require the necessary modifications of overland flow models to accommodate liquids with viscosity and density different than water. A completely developed spill model waits for coupling an overland flow code with a capable multiphase subsurface flow simulator. Building or finding such an integrated spill model and testing it is the task of the second stage of this work, which is to be accomplished in a future report.

To model a spill situation, an accurate description of the spill release or input to the land surface will be essential. This means that any generic description of spill events will be limited by lacking a description of exactly what was the pattern of spill release: slow escape through a hole in a tank or instantaneous bursting of a tank. The surface or overland flow will depend strongly on the input boundary conditions of the release.

An appropriate spill model should be able to predict the progress of reduction or loss of NAPL concentration near the surface, where it can be determined by remote sensing. In other words, a useful spill model should be capable of estimating the NAPL concentration near the surface as would actually be measured. Presently, it is not certain that the theory that applies to multiphase movement of NAPL in a porous subsurface material will produce an accurate description for the very first few millimeters of land surface. The disparity of land surface material properties contributes to this uncertainty.

Models that are available for describing the physics of liquid flow over surfaces and the infiltration into a variety of subsurface porous media are usually based on a continuum perspective that suggests that liquid flow is smooth flowing and predictable. However, overland flow and infiltration of certain liquids, depending on their density, viscosity, and interfacial tension, can be unstable and difficult-if not impossible-to predict. Certain liquids exhibit a fingering advance of flow across the land or into the ground. The theory of unstable liquid flows in the natural environment is just now under development, and few models exist. For liquids that are prone to exhibit unstable flow, this would make it impossible to rigorously predict spill spreading, at least in a verifiable way.

Considering the uncertainties imposed by the availability of material properties, acting in conjunction with inherent limitations in the mathematical flow theories, perhaps the best spill models that can be developed would be screening models that allow relative comparisons but are not expected to be accurate renditions of actual spills. 
At present, it is not possible to test or try out computer models that do not exist for treating spills. The next stage calls for coupling existing codes to see what predictions can be made relative to experimental confirmations. In particular, it seems prudent that simple experiments should be performed to gather data on the actual behavior of spills in relevant circumstances. For example, a spill on a roadway or on sand will likely behave quite differently. Data on typical spills could serve to help develop a correct theoretical viewpoint for future computer models.

A variety of simple modeling constructs were tested in this study. The usefulness of their predictions waits on confirming experimental data. Fortunately, the fluid dynamics of gravity currents has been supported with laboratory experiments. So far, representation of smooth flow over a surface by gravity currents has been tried in this study. The Green-Ampt model for infiltration was tried, and it was coupled with a gravity current to see if together these process models could mimic surface spreading. A simple fundamental capillary drainage model was devised to describe reduction of liquid content at the surface. An evaporation model was also tested. The problem of how to modify accurate analytical solutions of water infiltration to apply to NAPL was considered. An accurate analytical solution could be used to replace the Green-Ampt model or to improve its calibration for describing NAPL instead of just water.

It seems apparent, therefore, that the prediction of NAPL spill behavior as would be seen on the land surface is a work in progress. The challenge is to combine and couple the right computer models for overland and subsurface flow to describe spill phenomena. 


\subsection{References}

Acton, J. M., H. E. Huppert, and M. G. Worster. 2001. “Two-dimensional viscous gravity currents flowing over a deep porous medium.” Journal of Fluid Mechanics 440:359-380.

American Society of Civil Engineers (ASCE). 1996. "State-of-the-art review of modeling transport and fate of oil spills. " Journal of Hydraulic Engineering 122(11):594-609.

Arya, L. M. and J. F. Paris. 1981. "A physico-empirical model to predict soil moisture characteristics from particle-size distribution and bulk density data." Soil Science Society of America Journal 45:1023-1030.

Bear, J. 1972. Dynamics of Fluids in Porous Media. Dover Publications, New York.

Cavanaugh, T. A., J. H. Siegell, and K. W. Steinberg. 1994. "Simulation of vapor emissions from liquid spills." Journal of Hazardous Materials 38:41-63.

Clapp, R. B. and G. M. Hornberger. 1978. "Empirical equations for some soil hydraulic properties." Water Resources Research 14(4):601-604.

Dombrowski, H. S. and L. E. Brownell. 1954. "Residual equilibrium saturation of porous media." Flow Through Porous Media, Industrial and Engineering Chemistry 46(6): 1207-1219.

Dullien, F. A. L. 1992. Porous Media: Fluid Transport and Pore Structure. 2nd ed. Academic Press, Inc., San Diego.

Esteves, M., X. Faucher, S. Galle, and M. Vauclin. 2000. "Overland flow and infiltration modeling for small plots during steady rain: Numerical results versus observed values." Journal of Hydrology 228:265-282.

Fennema, R. J. and M. H. Chaudhry. 1990. "Explicit methods for 2-D transient free-surface flows." Journal of Hydraulic Engineering 116(8):1013-1034.

Fingas, M. F. 1995. "A literature review of the physics and predictive modeling of oil spill evaporation." Journal of Hazardous Materials 42:157-175.

Gee, G. W. and J. W. Bauder. 1986. "Particle-size analysis." In: Methods of Soil Analysis, Part 1 Physical and Mineralogical Methods, Agronomy Monograph No. 9 (2nd ed.), ed. A. Klute, American Society of Agronomy - Soil Science Society of America, Madison, Wisconsin.

Gvirtzman, H. and P. V. Roberts. 1991. "Pore scale spatial analysis of two immiscible fluids in porous media." Water Resources Research 27(6):1165-1176. 
Haverkamp, R., M. Vauclin, J. Touma, P. J. Wierenga, and G. Vachaud. 1977. "A comparison of numerical simulation models for one-dimensional infiltration." Soil Science Society of America Journal 41:285-294.

Hillel, D. 1998. Environmental Soil Physics. Academic Press, New York.

Huppert, H. E. 1982. "The propagation of two-dimensional and axisymmetric viscous gravity currents over a rigid horizontal surface." Journal of Fluid Mechanics 121:43-58.

Hussein, M., M. Jin and J. W. Weaver. 2002. "Development and verification of a screening model for surface spreading of petroleum." Journal of Contaminant Hydrology 57:281-302.

Jury, W. A., W. F. Spencer, and W. J. Farmer. 1983. "Behavior assessment model for trace organics in soil: I. Model description." Journal of Environmental Quality 12(4):558-564.

Kaluarachchi, J. J. and J. C. Parker. 1989. “An efficient finite element method for modeling multiphase flow." Water Resources Research 25(1):43-54.

Kao, C. S. and J. R. Hunt. 1996. "Prediction of wetting front movement during one-dimensional infiltration into soils." Water Resources Research 32(1):55-64.

Kapias, T., R. F. Griffiths, and C. Stefanidis. 2001. "ReactPool: A code implementing a new multicompound pool model that accounts for chemical reactions and changing composition for spills of water reactive chemicals." Journal of Hazardous Materials 81(1-2):1-18.

Leonard, J., E. Perrier, and G. de Marsily. 2001. "A model for simulating the influence of a spatial distribution of large circular macropores on surface runoff." Water Resources Research 37(12):3217-3225.

Lide, D. R. (ed.). 2002. CRC Handbook of Chemistry and Physics. 83rd ed. CRC Press, Boca Raton, Florida.

Lister, J. R. 1992. "Viscous flows down an inclined plane from point and line sources." J. Fluid Mech. 22: 631-653.

Mackay, D. and R. S. Matsugu. 1973. "Evaporation rates of liquid hydrocarbon spills on land and water." The Canadian Journal of Chemical Engineering 51:434-440.

Marshall, T. J. and J. W. Holmes. 1979. Soil Physics. Cambridge University Press, Cambridge.

McBride, J. F., C. S. Simmons and J. W. Cary. 1992. "Interfacial spreading effects on one-dimensional organic liquid imbibition in water-wetted porous media." Journal of Contaminant Hydrology 11:1-25.

Mercer, J. W. and R. M. Cohen. 1990. "A review of immiscible fluids in the subsurface: Properties, models, characterization and remediation.” Journal of Contaminant Hydrology 6:107-163. 
Miller, C. T., G. Christakos, P. T. Imhoff, J. F. McBride, and J. A. Pedit. 1998. "Multiphase flow and transport modeling in heterogeneous porous media: Challenges and approaches." Advances in Water Resources 21(2):77-120.

Mohanty, B. P., P. J. Shouse, D. A. Miller and M. T. van Genuchten. 2002. "Soil property database: Southern Great Plains 1997 Hydrology Experiment.” Water Resources Research 38(5). Article 1047.

National Academy of Science (NAS). 2003 Oil in the Sea III: Inputs, Fates, and Effects. National Academies Press, Washington, D.C.

Natural Resources Conservation Service (NRCS). Soil Survey. Pub. U.S. Department of Agriculture Natural Resources Conservation Service, Washington, D.C.

Nemes, A., M. G. Schaap, F. J. Leij, and J. H. M. Wosten. 2001. "Description of the Unsaturated Soil Hydraulic Database UNSODA Version 2.0.” Journal of Hydrology. 251:151-162.

Neuman, S. P. 1976. "Wetting Front pressure head in the infiltration model of Green and Ampt." Water Resources Research 12(3):564-566.

Oscarson, J. L., R. L. Rowley and W. V. Wildung. 2003. Design Institute for Physical Properties Database. Available: Brigham Young University, Provo, Utah.

Parker, J. C. 1989. "Multiphase flow and transport in porous media." Reviews of Geophysics 27(3):311-328.

Parlange, M. B., A. T. Cahill, D. R. Nielsen, J. W. Hopmans, and O. Wendroth. 1998. "Review of heat and water movement in field soils." Soil and Tillage Research 47:5-10.

Philip, J. R. 1969. "Theory of infiltration.” Adv. Hydrosciences 5:215-305.

Rawls, W. J., L. R. Ahuja, D. L. Brakensiek, and A. Shirmohammadi. 1993. "Infiltration and soil water movement." Chapter 5 in Handbook of Hydrology, ed. D. R. Maidment, McGraw-Hill, Inc., New York.

Reible, D. D., T. H. Illangasekare, D. V. Doshi, and M. E. Malhiet. 1990. "Infiltration of immiscible contaminants in the unsaturated zone." Groundwater 28(5):685-692.

Reinke, P. H. and L. M. Brosseau. 1997. "Development of a model to predict air contaminant concentrations following indoor spills of volatile liquids." Annals of Occupational Hygiene 41(4):415-435.

Schaap, M. G., F. J. Leij, and M. T. van Genuchten. 2001. "ROSETTA: A computer program for estimating soil hydraulic parameters with hierarchical pedotransfer functions." Journal of Hydrology 251:163-176.

Schwarzenbach, R. P., P. M. Gschwend, and D. M. Imboden. 1993. Environmental Organic Chemistry. John Wiley \& Sons, Inc., New York. 
Sturman, P. J., P. S. Stewart, A. B. Cunningham, E. J. Bouwer, and J. H. Wolfram. 1995. "Engineering scale-up of in situ bioremediation processes: A review." Journal of Contaminant Hydrology 19:171-203.

Van Den Berg, F., R. Kubiak, W. G. Benjey, M. S. Majewski, S. R. Yates, G. L. Reeves, J. H. Smelt, and A. M. A. Van Der Linden. 1999. "Emission of pesticides into the air." Water, Air, and Soil Pollution 115:195-218.

Warrick, A. W., D. O. Lomen, and A. Islas. 1990. “An analytical solution to Richards equation for a draining soil profile." Water Resources Research 26:253-258.

Warrick, A. W., A. Islas, and D. O. Lomen. 1991. "An analytical solution to Richards' equation for time-varying infiltration." Water Resources Research 27(5):763-766.

Weaver, J. W., R. J. Charbeneau, and B. K. Lien. 1994. "A screening model for nonaqueous phase liquid transport in the vadose zone using Green-Ampt and kinematic wave theory." Water Resources Research 30(1):93-105.

White, M. D., M. Oostrom, and R. J. Lenhard. 1995. "Modeling fluid flow and transport in variably saturated porous media with the STOMP simulator. 1. Nonvolatile three-phase model description." Advances in Water Resources 18(6):353-364.

White, M. D. and M. Oostrom. 2000. STOMP Subsurface Transport over Multiple Phases. Version 2. Theory Guide. PNNL-12030, Richland, Washington.

Woolhiser, D. A. and J. A. Liggett. 1967. "Unsteady, one-dimensional flow over a plane - The rising hydrograph." Water Resources Research 3(3):39-71.

Yates, S. R., D. Wang, S. K. Papiernik, and J. Gan. 2002. "Predicting pesticide volatilization from soils." Environmetrics 13:569-578.

Zhang, W. and T. W. Cundy. 1989. "Modeling of two-dimensional overland flow." Water Resources Research 25(9):2019-2035. 